\title{
On the histological diagnosis and prognosis of malignant melanoma
}

\author{
ARNOLD LEVENE \\ Hunterian Professor, Royal College of Surgeons, and Department of Histopathology, The Royal Mursden \\ Hospital, Fulham Road, London SW3, UK
}

SUMMARY This review deals with difficulties of diagnosis in cutaneous malignant melanoma encountered by histopathologists of variable seniority and is based on referred material at The Royal Marsden Hospital over a 20-year period and on the experience of more than two-and-a-half thousand cases referred to The World Health Organisation Melanoma Unit which I reviewed when chairman of the Pathologists' Committee. Though there is reference to the differential diagnosis of primary and metastatic tumour, the main concern is with establishing the diagnosis of primary melanoma to the exclusion of all other lesions. An appendix on recommended diagnostic methods in cutaneous melanomas is included.

Among the difficult diagnostic fields in histopathology melanocytic tumours have achieved a notoriety. Accurate diagnosis, however, is of major clinical importance for the following reasons:

1 The management of the primary lesion is principally by surgical excision with a large margin of normal appearing skin. The consequences of over-diagnosis are those of major disfiguring surgery and its morbidity.

2 The penalty for under-diagnosis or inadequate excision of a primary melanoma is local recurrence, which carnies a mortality greater than that of an adequately excised primary lesion.

3 With an incomplete diagnosis, that is, one in which significant observations have been omitted from the pathologist's report, the patient may be subjected to inappropriate therapy.

4 Cutaneous melanomas are a group of malignancies in which there is a premium on diagnosis at the in situ and early invasive phases. There is a definite association between progressive depth of invasion and a worsened prognosis.

The difficulties peculiar to the histological diagnosis of melanomas are the differentiation of benign and malignant melanocytoma and the differentiation of malignant melanomas from all other non-melanocytic tumours both benign and malignant. In practice, the former provides the more formidable problems. Histological criteria of malignancy are entirely dependent on the observed behaviour associated with a given cellular pattern. A cellular proliferation is not to be labelled malignant because it 'looks nasty'. Thus, until the critical evaluation of the 'malignant melanoma of childhood' by Spitz (1948) the naevus with which this investigator's name is associated was reckoned among the malignancies on histological grounds.

\section{Naevus and melanoma cells}

A major problem in the diagnosis of primary melanoma is its distinction from innocent proliferations of naevus cells. Both benign and malignant melanocytic proliferations are composed of groups of cells, of which only a small proportion may have recognisable, normal counterparts. At the light microscope level they may or may not exhibit melanogenesis. They are associated with a variable degree of distortion or apparent destruction of epidermis and, on occasion, panniculus and are unencapsulated.

The novice in pathology is particularly puzzled by the term naevus cell since it is applied to nests of cells at the dermoepidermal junction, intradermal cells, and intraepidermal cells, and there are apparent differences between naevus cells in each of these different situations. Only considerable experience of the wide range of the cytoplasmic and nuclear configurations and the cellular groupings to be encountered in the junctional compound and intradermal naevi will provide the familiarity with the naevus melanocyte which is indispensable as a 
yardstick of innocence against which malignant melanocytic proliferation is gauged. Indeed, individual fields of naevus and melanoma may be indistinguishable on cytological grounds in good haematoxylin and eosin preparations, and a distinction between the two in such cases ultimately rests on an assessment of the overall growth pattern. Familiarity with the structure of naevi and their variations is the key to most problems associated with the diagnosis of melanoma. Excellent accounts of the appearances of naevi in the different stages of their developments are given by Shaffer (1955) and Lund and Stobbe (1949).

Naevus cells (Fig. 1) may be large or small, with ill-defined cell boundaries or epithelioid and spindle cell form with clear or acidophilic cytoplasm. They may be single or multinucleated, with or without a nucleolus (the nucleolus is conspicuous in the Spitz naevus). Pigmentation may be present but if absent the cells may be identified from their growth patterns, grouping, and relation to other cutaneous structures. Mitoses are rarely seen in junctional naevus and its variants and are found in the junctional zone only, never in intradermal nests. Mitoses are a common feature of the Spitz naevus, and innocent behaviour of this lesion has been associated with abnormal mitosis (Kernen and Ackerman, 1960). A common, probably artefactual, nuclear anomaly seen in naevus cells is the presence of intranuclear vacuolation. Some consider simple lentigo (Fig. 2) an incipient naevus.

Melanoma cells are in general larger than naevus cells and likely to contain finely dispersed melanin even away from the immediate subepidermal region. The large nucleus may contain a prominent acidophilic nucleolus and show mitotic activity. One must on occasion be prepared to diagnose melanoma in almost the entire absence of melanin production and in the presence of bizarre cellularity, which bears no resemblance to normal melanocytes. Bizarre melanoma cells are seen after irradiation particularly, but may be present in untreated melanomas or their metastases as a spontaneous development.

Both naevi and melanomas are likely to show a variety of cell types and growth pattern in the same tumour. In some naevi there is a gradation from epidermis to the depth of the lesion, plumper, more pigmented cells being superficial while the depths are occupied by sclerosing cellular tissue exhibiting the lames foliacées stressed by Masson (1951). Sometimes this alteration in the depth of a lesion consists of a crowding of cells with less cytoplasm than the superficial component. Either type of appearance is referred to as maturation. Since a similar growth pattern may be seen in melanoma its presence is not an absolute criterion of innocence.

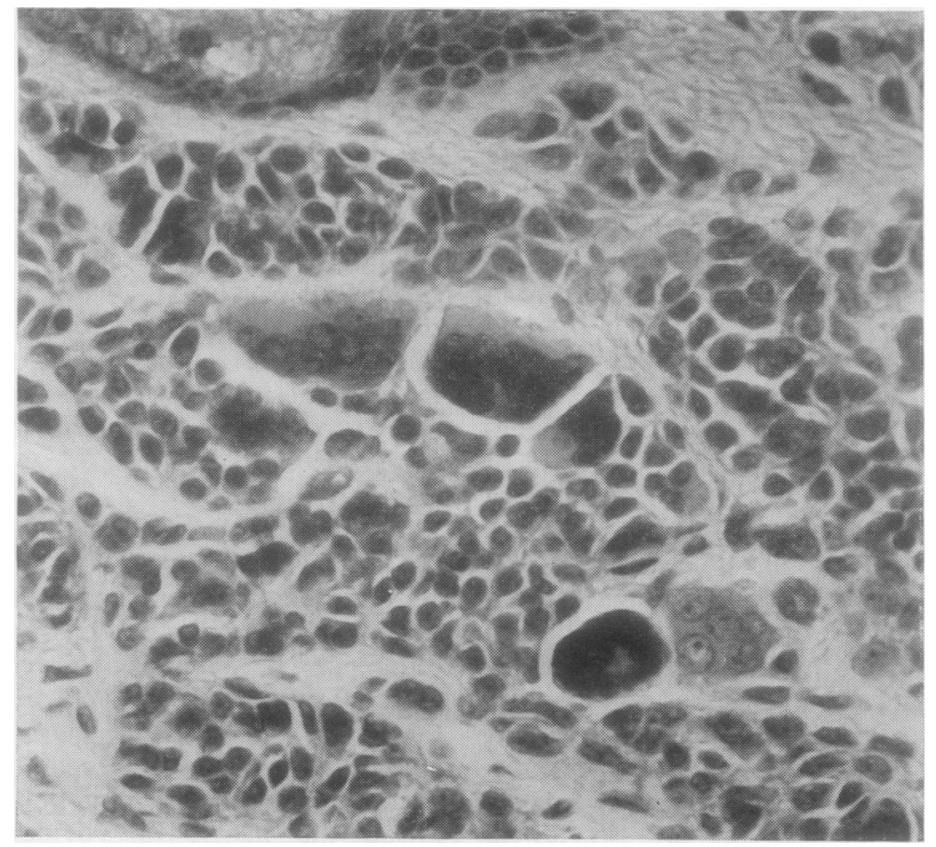

Fig. 1 Intradermal naevus cells with multinucleated forms. 


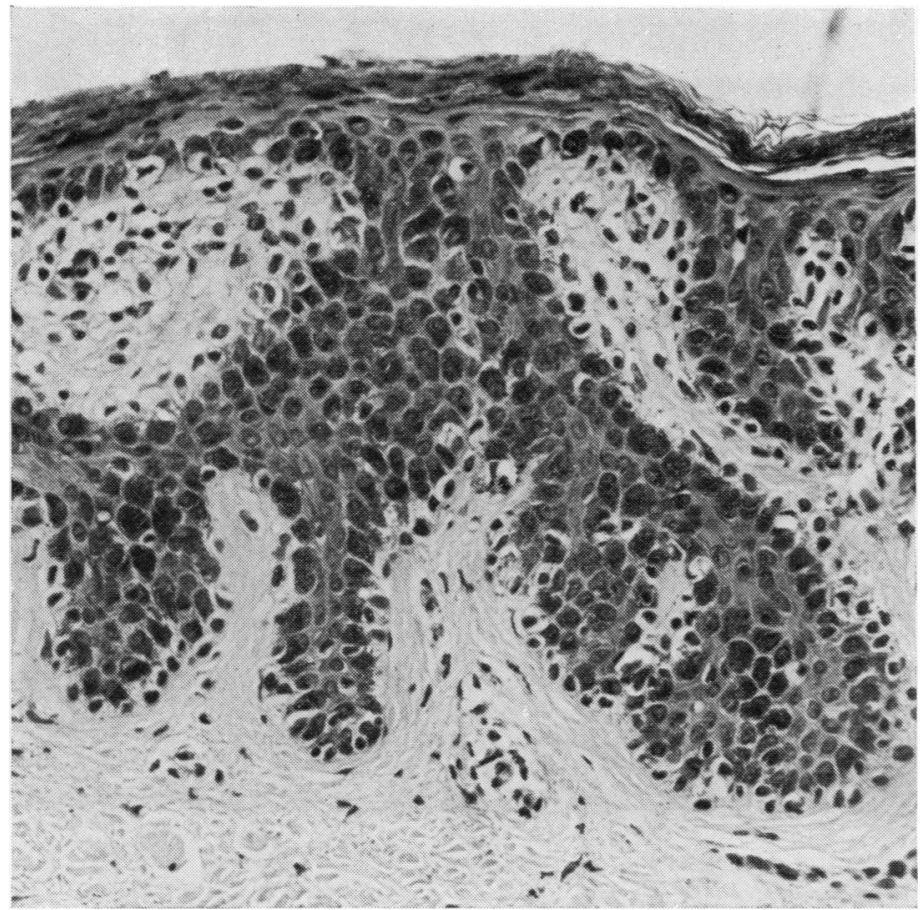

Fig. 2 'Thumblike' hyperpigmented papillomatosis is seen in benign lentigo.

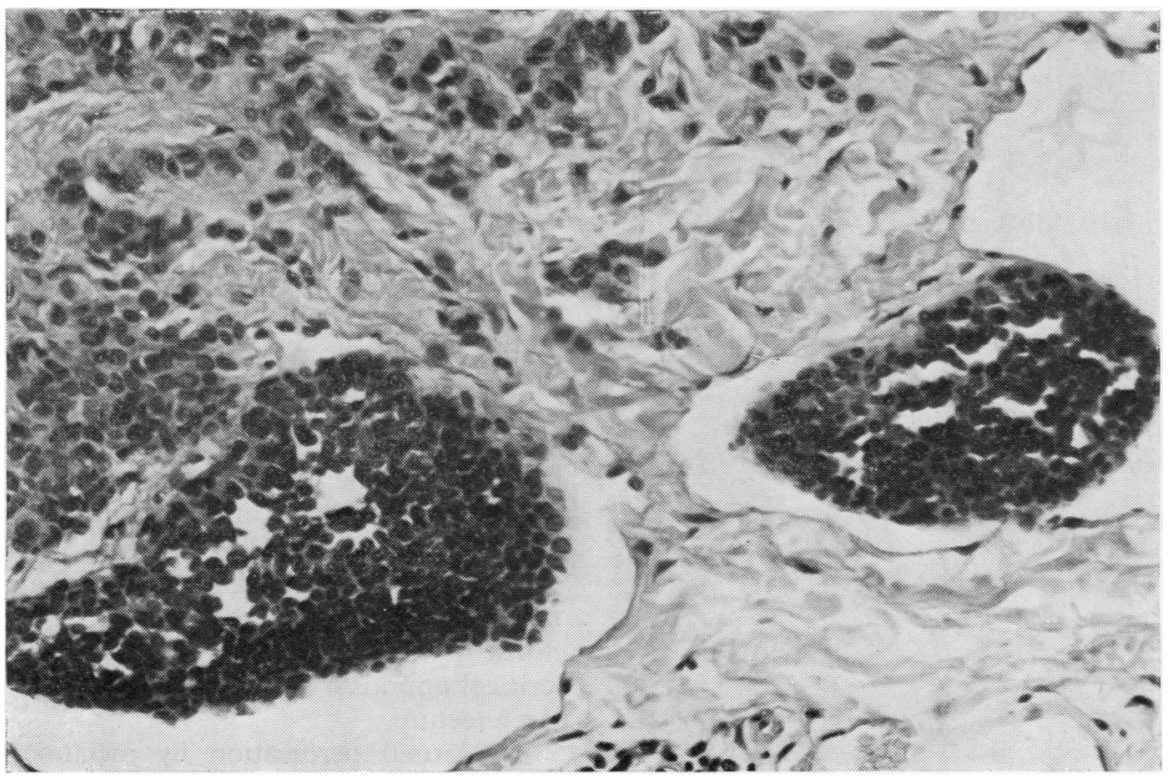

Fig. 3 Occasionally, in an intradermal naevus, intravascular naevus cells are found. Whether these appearances are truly those of lymphatic infiltration or simulated by a bulging of a naevus cell collection beneath an intact vascular endothelium they have no clinical significance. 


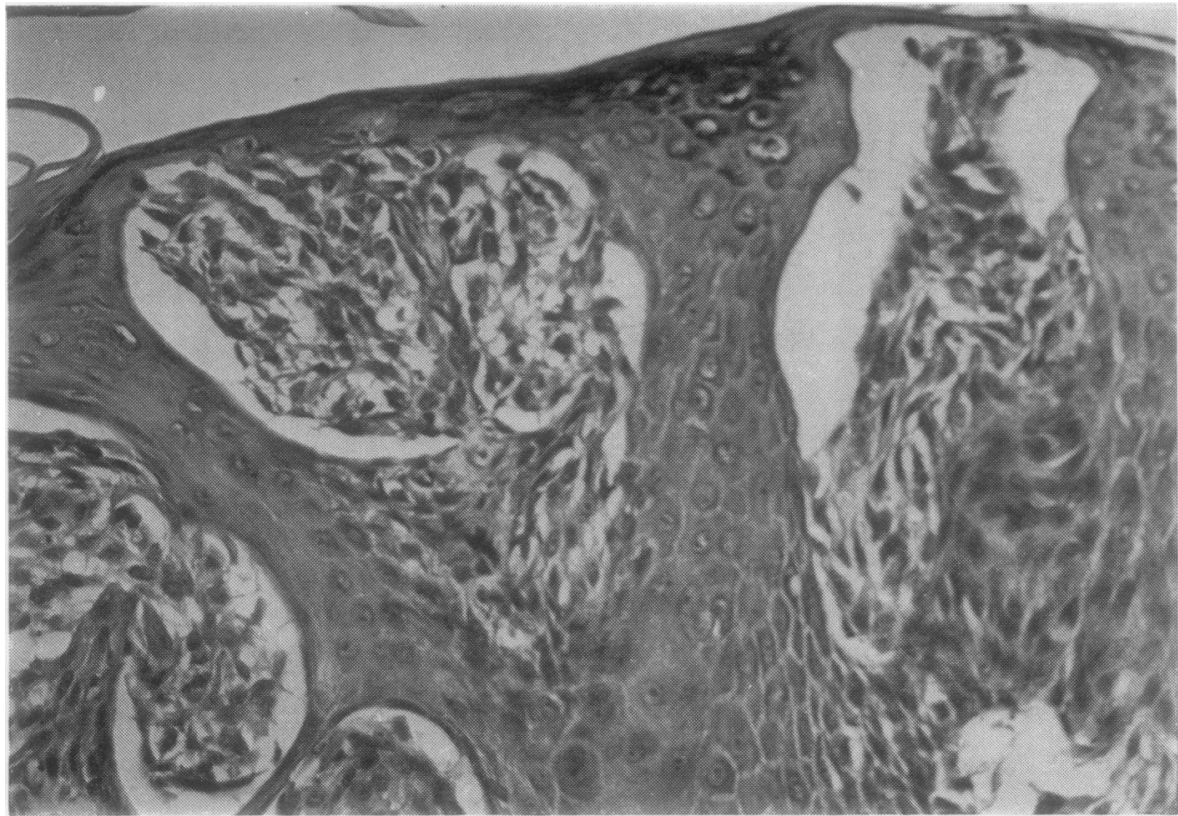

Fig. 4 From a compound naevus of childhood exhibiting the phenomenon of transepidermal elimination of naevus cell nests.

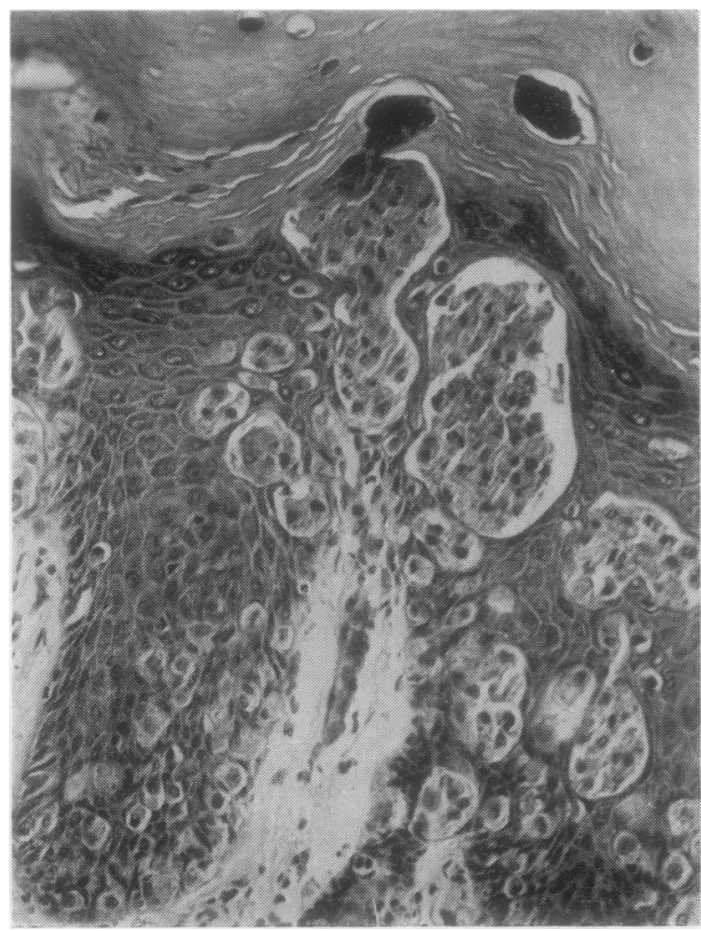

Fig. 5 Typical malignant junctional change. Melanin pigmentation of keratin, also well shown here, is seen in naevi and has no discriminatory value.
Similarly, the distribution of pigment in both naevus cell naevi and a malignant melanoma is mainly superficial in relation to the whole lesion, but a patchy distribution in the depth is not a reliable indicator of malignancy. Naevus cell extension into fat, muscle, and lymphatics (Fig. 3) is not an indication of malignancy.

\section{Intraepidermal melanocytes}

In the epidermis, changes that are diagnostic of primary melanoma may be found. Melanocytes, whether normal, transformed but benign (naevus cells), or malignant, may be differentiated from the keratinocytes at low powers of the microscope. In the normal basal situation they possess an artefactual perinuclear halo, which characterises them as clear cells. In melanocytic naevi and melanoma, melanocytes may come to occupy a suprabasal position in the prickle-cell layer and beyond (Figs 4 and 5). But whereas this is fairly constantly observed in those melanomas in which an epidermal genesis is apparent, it is so uncommon in naevi other than those in infants that it should lead to the most critical appraisal of the lesion's cytology and genera architecture.

Epidermal permeation by melanocytes may be either as single cells or as nests. Where there is epidermal destruction by nests of melanocytes the lesion is clearly malignant, but single cell permeation 


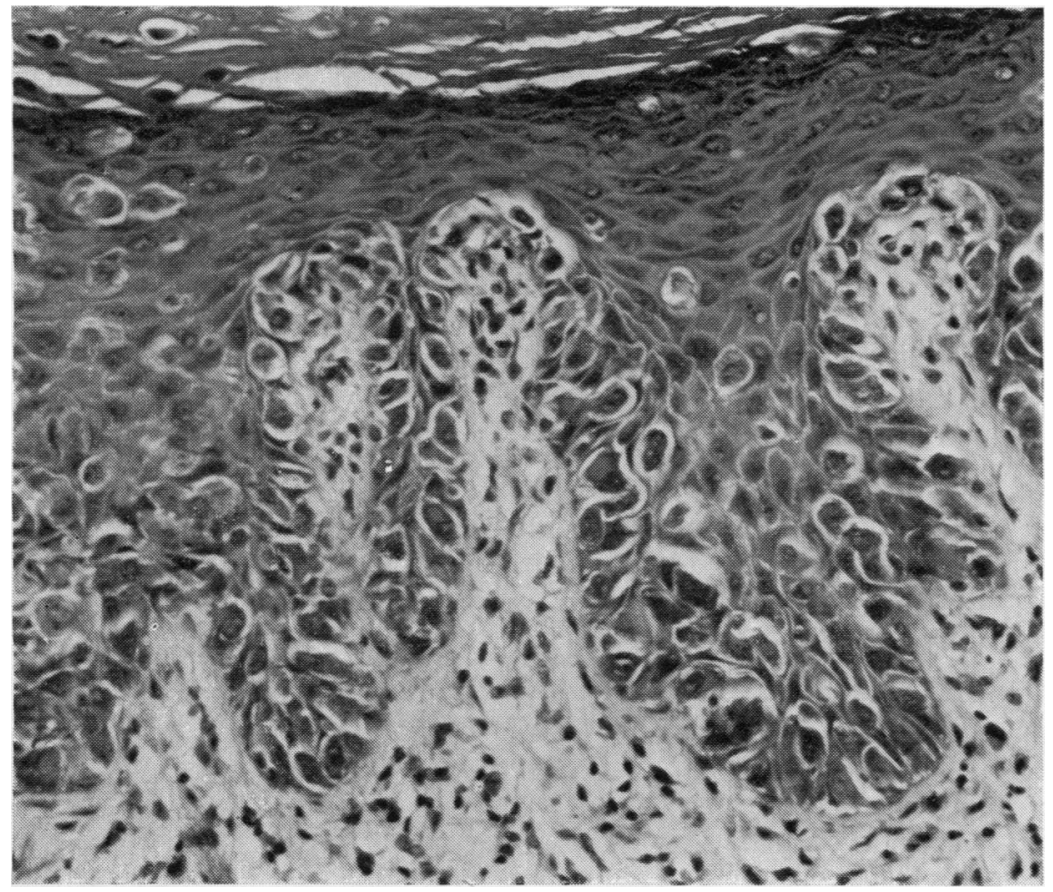

Fig. 6 Paget's disease of perianal skin.

may present a problem of diagnosis. Thus, the permeating cells may not be melanocytes. Intraepidermal melanocytes have to be distinguished from 'high-level, clear cells' or Langerhans cells, secondary epidermotrophic carcinoma, and intra- epidermal mucin-secreting carcinoma-Paget's disease. The former, for many years of debatable status and often considered to be effete melanocytes, are thought by Breathnach (1968) to be of histiocytic type. The nuclei are small, regular, normochromatic,

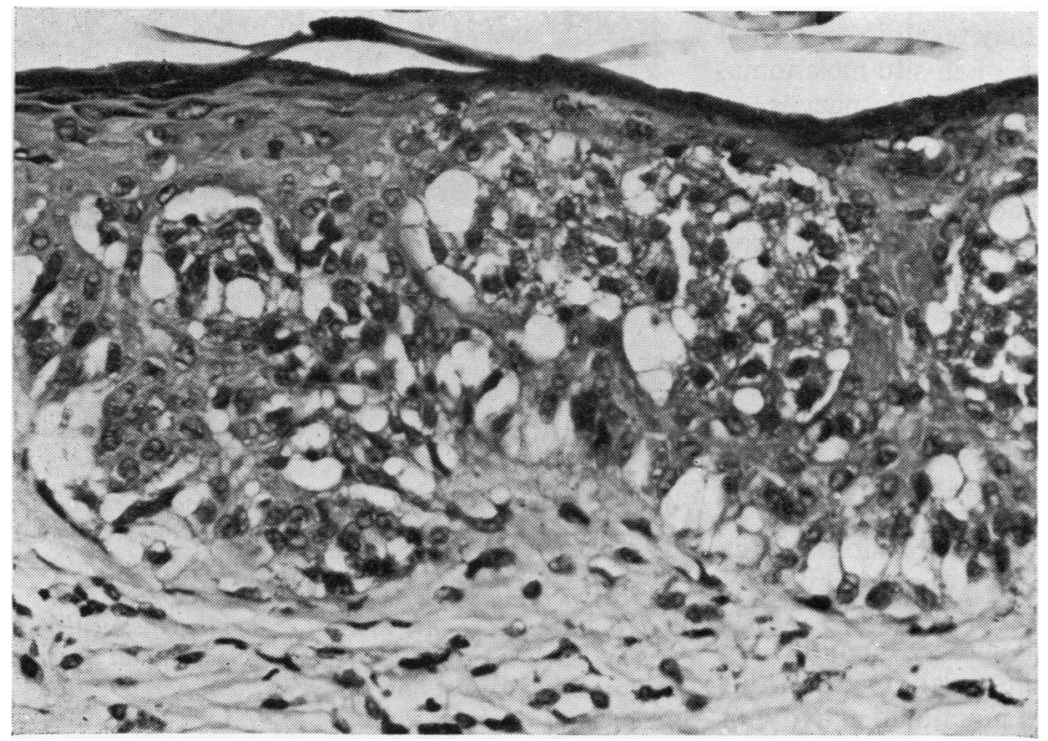

Fig. 7 Kornberg and Ackerman (Archives of Dermatology, 1975, 111, 1588-1590) described the remarkable appearances in the skin after the therapeutic shaving of a naevus, an attempt at ablation not used, to my knowledge, outside of the USA. The appearances simulate in situ melanoma. (By kind permission of Dr Ackerman.) 
and never in mitosis. The cytoplasm is invariably clear and contrasts with the appearance of naevus cells and melanoma cells. If there is a suspicion that scattered, ballooned, intraepidermal cells are Paget cells the appropriate mucin stains (mucicarmine, diastase-PAS) should be used for confirmation. This is a very practical problem since extramammary Paget's disease (Fig. 6) may simulate lentigo maligna in particular. Though melanin may be present within Paget cells, mucin is invariably absent from melanoma. Secondary intraepidermal carcinoma, not of Paget type, may also simulate intraepidermal melanoma.

It may be extremely difficult to categorise intraepidermal melanocytes as benign or malignant. The diagnostic problem is greatest in those cases where the qualitative nature of the junctional change is equivocal because of histological artefact. Solution of the dilemma comes from fitting the epidermal appearances together with the complete clinical picture. In infancy and in adults after solar exposure in particular, in an otherwise junctional or compound naevus there may also be associated single cell (melanocytic) permeation of the epidermis to a limited degree. The cells, however, lack the stigmata of malignancy, though occasionally these cells are observed in mitosis. Infancy and 'non-malignant' cytology in a lesion from an adult sway one towards the diagnosis of a simple naevus.

Injury to junctional naevus from:

1 attempts at ablation of the naevus by therapeutic shaving, an 'office' procedure in the USA (Fig. 7),

2 cosmetic shaving of the legs in women, or

3 sunbathing

( 2 and 3 may be combined) may result in epidermal changes which simulate those of in-situ melanoma, while deeply the invasive portion of the naevus, or its remains, are unaffected. In these situations, the history should alert the pathologist-in the case of (1) it is crucial-while in (2) and (3) the melanocytes migrating singly in the epidermis, though atypical, lack obvious malignant features. Assessment in these cases is admittedly difficult. Where doubt persists, the pathologist and clinician must face the limitations of the histological method and consent to a guarded diagnosis with regular follow-up for some years. However, the important matter is whether invasive melanoma is present or not. Where the diagnosis lies between junctional naevus and some form of pre-invasive melanoma, complete excision of the lesion is curative for either and the likelihood of metastasis from the latter remote.

There may also be clear cell activity in epithelium overlying metastatic malignant melanoma, and this may be indistinguishable from junctional activity, of innocent or borderline status, though I have not observed continuity between these changes and the underlying melanoma. Such an appearance may be of significance in connection with a diagnosis of nodules which appear in the neighbourhood of a melanoma-satellite nodules-frequently after excision of the lesion. These may be fresh primary tumour but more often are banal secondary deposits, with the confusing appearance of simple junctional change overlying the metastatic tumour.

\section{Junctional change}

The term junctional change means melanocytic proliferation in the junctional zone between the dermis and the epidermis with its derivatives. There is no implication of innocence or malignancy in the

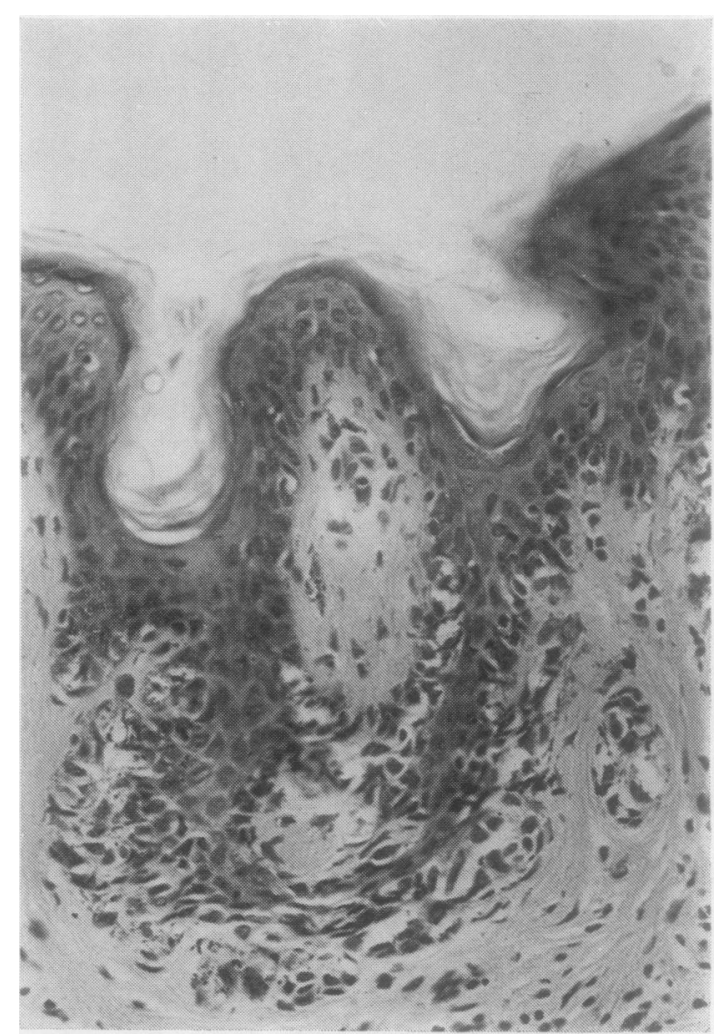

Fig. 8 A pre-pubic skin blemish with no recent changes in appearance, histologically a compound naevus. The junctional change lacks malignant quality, but the appearances alarmed the referring pathologist. The solution to uncertainty is not to issue an equivocal report but to seek further advice. Then, should uncertainty still persist, it should be stated in the report that the lesion is of doubtful status. 


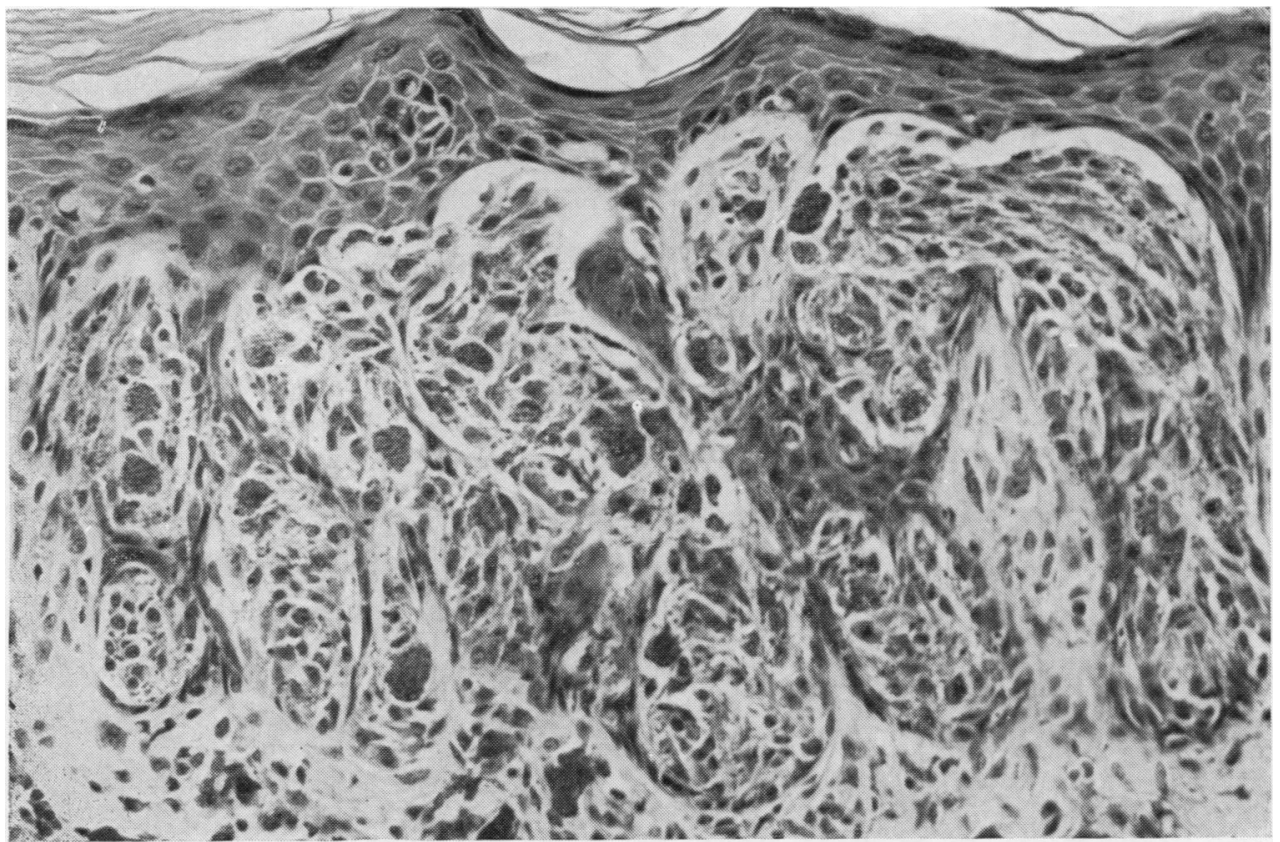

Fig. 9 Pre-invasive, superficial, spreading melanoma. In the lesion of debatable innocence, the finding of mitoses, nuclear aberrations or prominent nucleoli and the continuous nature of the junctional change are factors weighting a decision in favour of malignancy.

use of the term since it is virtually a constant feature of naevocytic naevi at some stage of their development. A good account of benign junctional activity, that is, the changes associated with junctional compound and intradermal naevi, is provided by Lund and Kraus (1962). In its incipient phase, a focal multiplication of clear cells is observed in the basal layer of the epidermis (Fig. 8). Such collections are referred to as theques. Kopf and Andrade (1963) have suggested that an aggregation of five melanocytes is the minimum to warrant this designation, but they may contain hundreds of melanocytes. They are situated in and on the rete ridges. The older literature deals with the phenomenon of Abtropfung, by which the melanocytes enter the dermis and with some change of shape undergo a change of name to naevus cells (Unna, 1896).

In malignant junctional change, the commonest concomitant of malignant melanoma, the appearances are extremely variable but may be distinguished from the innocent counterpart by a combination of cytological and microarchitectural appearances. Most primary melanomas have obvious cytological malignant characteristics, and in these the in situ lesion (Fig. 9) is characterised by larger cells with voluminous, somewhat dusky eosinophilic cytoplasm and larger hypochromatic nuclei with prominent nucleolus. Yet there are cases in which the differences between the innocent and malignant melanocytes may be absent or of negligible or debatable degree. The situation is analogous to the case of verrucous carcinoma arising in a squamous mucosa where it is the growth pattern, the microarchitecture, of the epithelial proliferation coupled with the presence of minimal atypia which provide the important diagnostic features.

It must be recognised that, as far as the intensity and degree of destruction of the epidermis by junctional change is concerned, there may be little or nothing to distinguish innocence from malignancy. Junctional activity tending to produce balls of cells bulging into both dermis and epidermis inevitably distorts, compresses, and frays the squamous epithelium. With innocent junctional change these alterations are predominantly on the rete ridges whereas in the in situ melanoma there is proliferation of melanocytes between as well as upon the ridges (Fig. 10). Hair growth in a tumour does not exclude the diagnosis of malignancy, since melanoma may arise in association with a compound or intradermal naevus and the malignancy may involve only part of the lesion. 


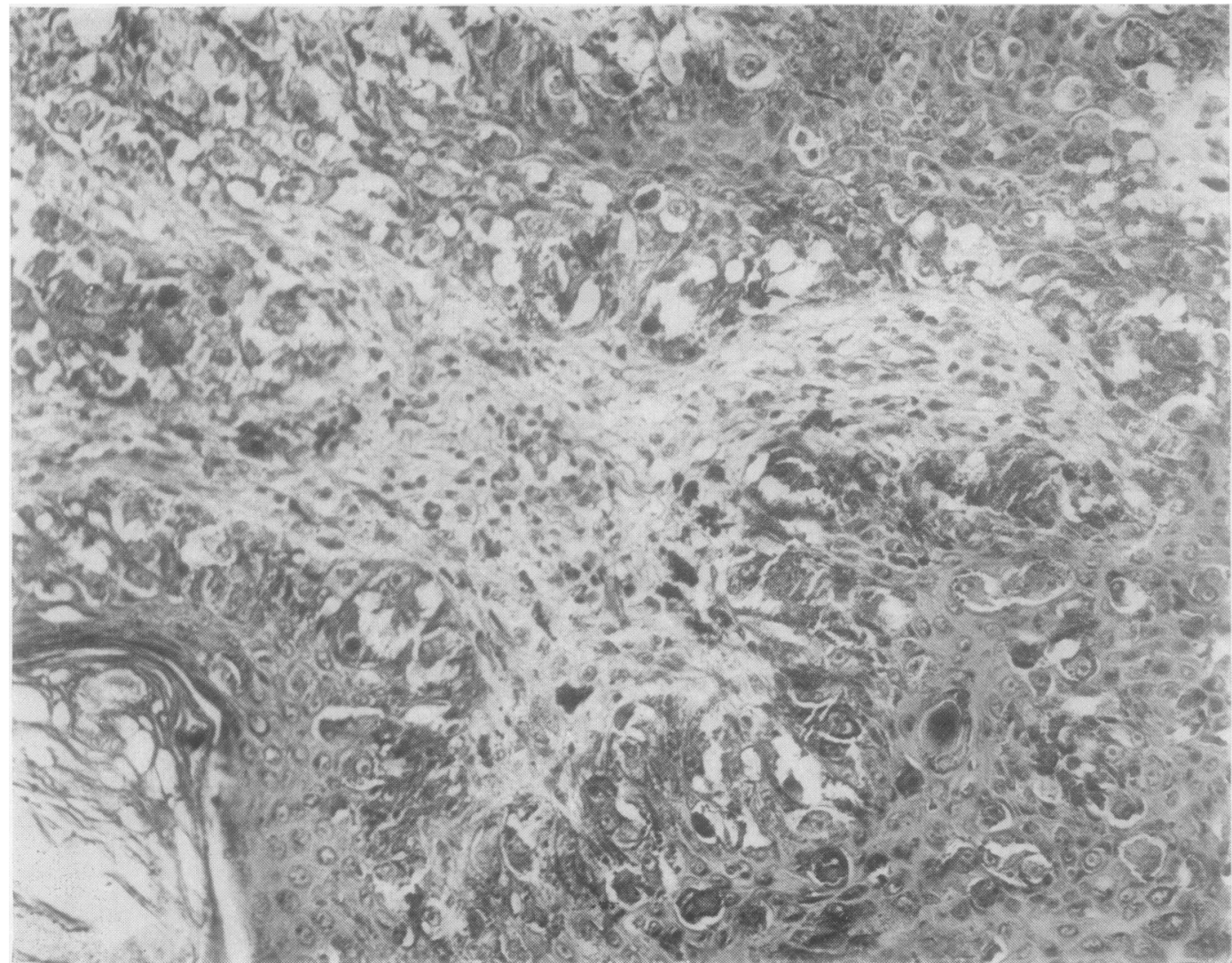

Fig. 10 Malignant junctional change. Apart from its tendency to be associated with extensive permeation of the suprabasal epidermis it tends to be continuous along the rete ridges.

\section{'Pre-invasive malignant melanoma'}

Two types of malignant junctional change may be described corresponding to two main recognised clinical entities. One is found in the superficial spreading type of melanoma, the common melanoma of the female calf and of the trunk of both sexes. The second type of junctional change is called lentigo maligna (synonym: Hutchinson's freckle). This occurs principally on the exposed skin of the head and also on the conjunctiva and the non-exposed skin and mucosa. Both superficial spreading and lentigo maligna melanoma consist of a focus of dusky nodularity and induration in association with pigmentation of the adjacent skin. The latter may be of minimal extent in superficial spreading melanoma and may show, in addition to brown and black areas, red, white, and bluish-grey changes. The Hutchinson freckle is similar but, whereas the play of colours associated with spontaneous regression may be a feature of superficial spreading melanoma, it is invariable in lentigo maligna.

The junctional change characterising superficial spreading melanoma (Fig. 11) is composed of fairly uniform cells with abundant cytoplasm which is dusted with melanin and nuclei resembling those of naevus cells or larger hyperchromatic forms with one or two nucleoli. Early in its development there is a close resemblance between this type of melanoma and junctional naevus since the grouping of the cells is similar, and both infiltrate the dermis. In malignant junctional change, though, there is invasion of the epidermis by single cells and nests ultimately with a destructive effect.

In the earliest manifestations of this type of superficial melanoma, dermal invasion is absent, and the condition may be referred to as melanoma in situ. Histologically, it bears considerable resemblance to Paget's disease of the nipple. When a melanoma is seen to arise by this mode of histogenesis it may be 


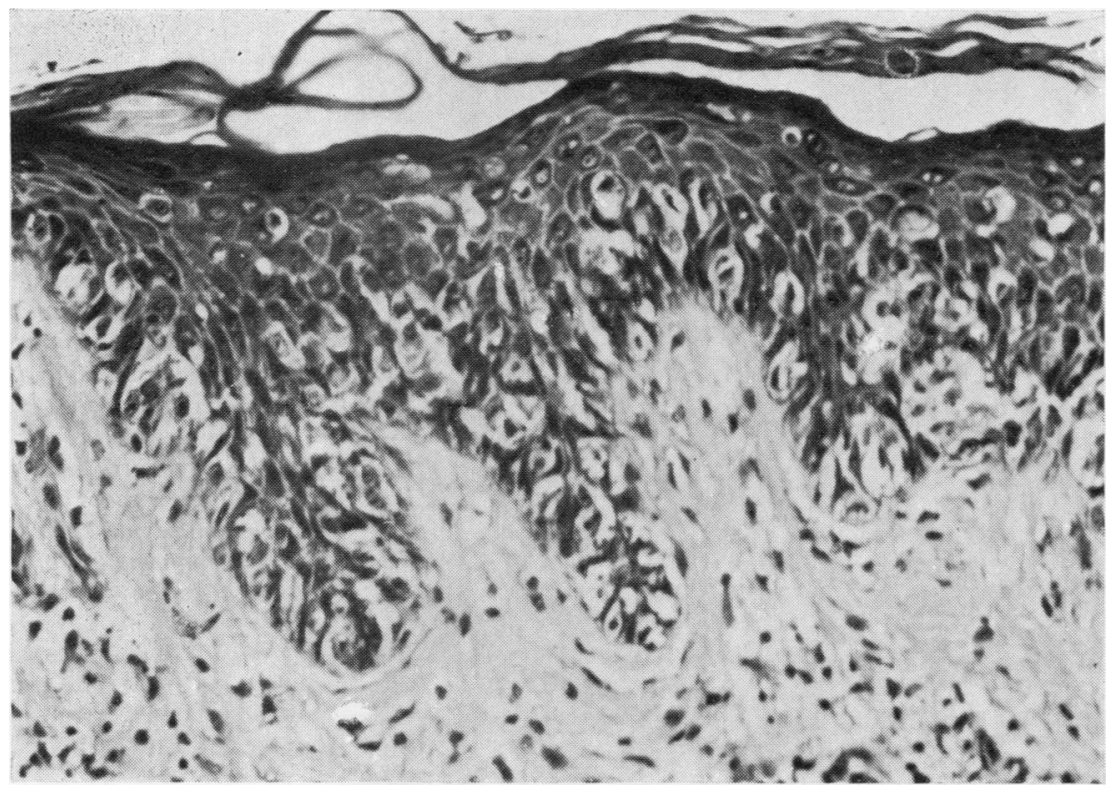

Fig. 11 The

continuous nature of the junctional change in the basal layer of the epidermis is characteristic of the superficial spreading variant of in situ melanoma.

referred to histologically as being of superficial spreading type. Further epidermal changes occur beyond the region of malignant junctional change in superficial spreading melanoma, and there is hyperpigmentation of the basal layer of the epidermis associated on occasion with the scattered presence of abnormal melanocytes.

In lentigo maligna, studies of the pigmented or depigmented zones (Clark and Mihm, 1969) reveal foci of increased numbers of basal melanocytes with occasional atypical forms. There is great variation of the melanocytes with bloated cytoplasm, giant and multinucleated cells, and hyperpigmentation. The melanocytes of lentigo maligna (Fig. 12) are charac- terised by a spectrum of continuous variability from hyperchromatic normality to gross abnormality and in isolated fields do not differ from senile lentigo (Fig. 2). The normal basal keratinocyte/melanocyte relationship is replaced by a continuous monolayer of large atypical melanocytes which also exhibit nesting as in other forms of junctional change. Junctional change in adnexa is seen particularly in lentigo maligna. Suprabasal type permeation of 'Pagetoid' type is uncommon. In general, superficial spreading melanoma cells in the epidermis are characterised by a uniformity of appearance and nest formation while lentigo maligna cells are bloated and pleomorphic with a tendency to palisade

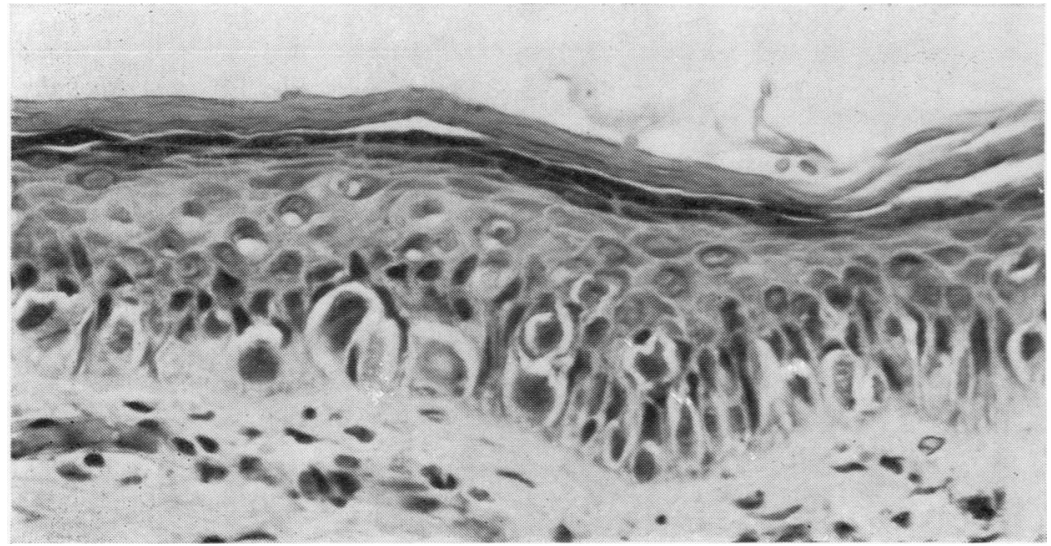

Fig. $12 A$ wide range of cellular pleomorphism characterises the atypical melanocytosis of lentigo maligna. 


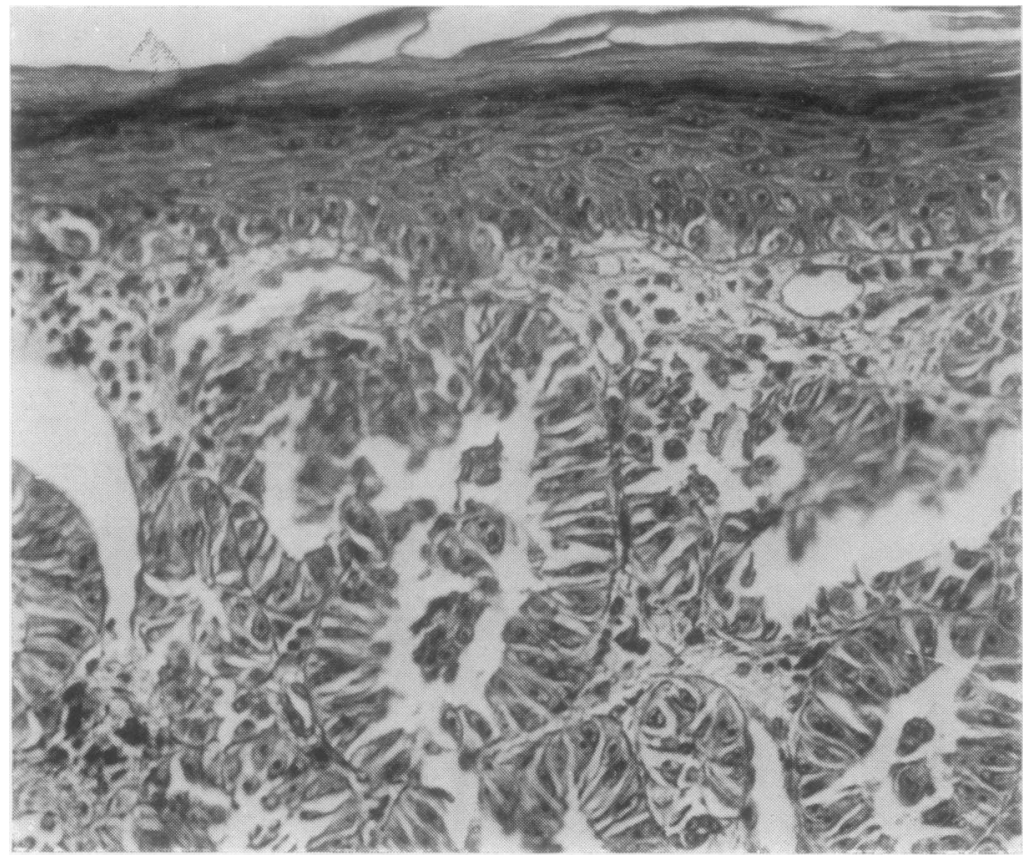

Fig. 13 A papillary acinar pattern seen in a primary melanoma. The tumour was mainly achromic, but the detection of dark granules, which proved Fontana positive, and more usual appearances of melanoma in other fields led to confirmation of the diagnosis.

in the basal layer. In the dermis of lentigo maligna, phagocytosis of pigment, reactive lymphocytes, and macrophages together with solar degeneration of collagen complete the picture.

The early stage of subungual melanoma has features in common with both lentigo maligna and superficial spreading melanoma but there is a conspicuous difference in the prominent dendritic nature of the basally placed malignant melanocytes. The infiltrating phase lacks characteristic features.

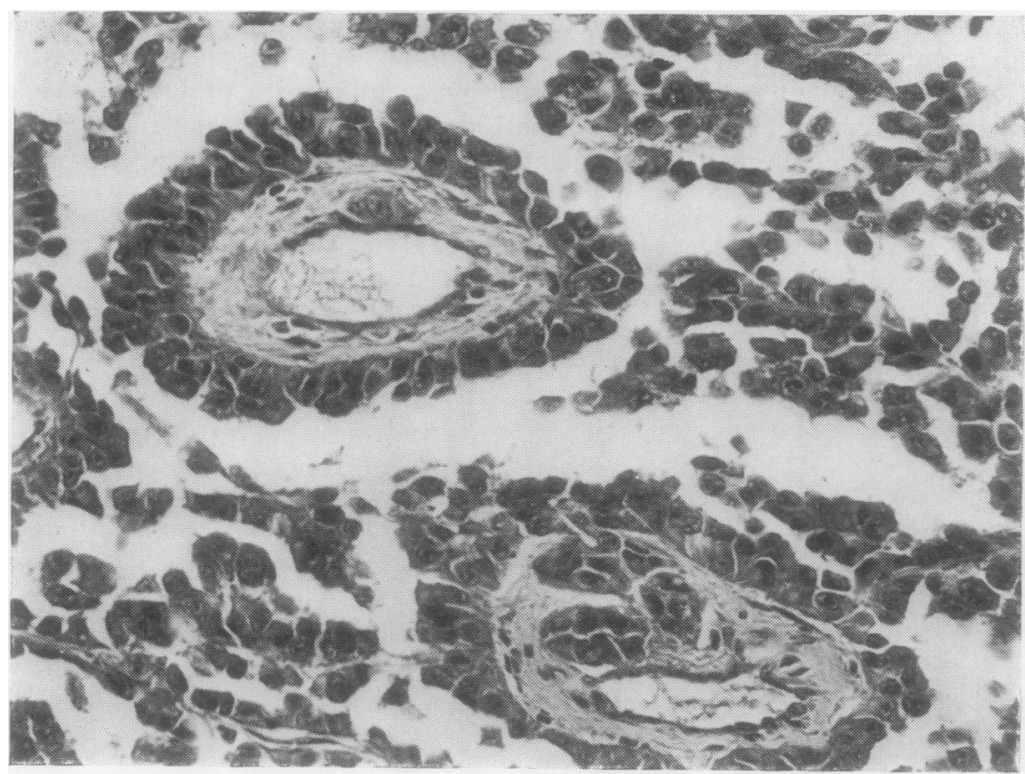

Fig. 14 A perithelial, pseudopapillary growth pattern of melanoma simulating, for example, renal or thyroid carcinoma. 


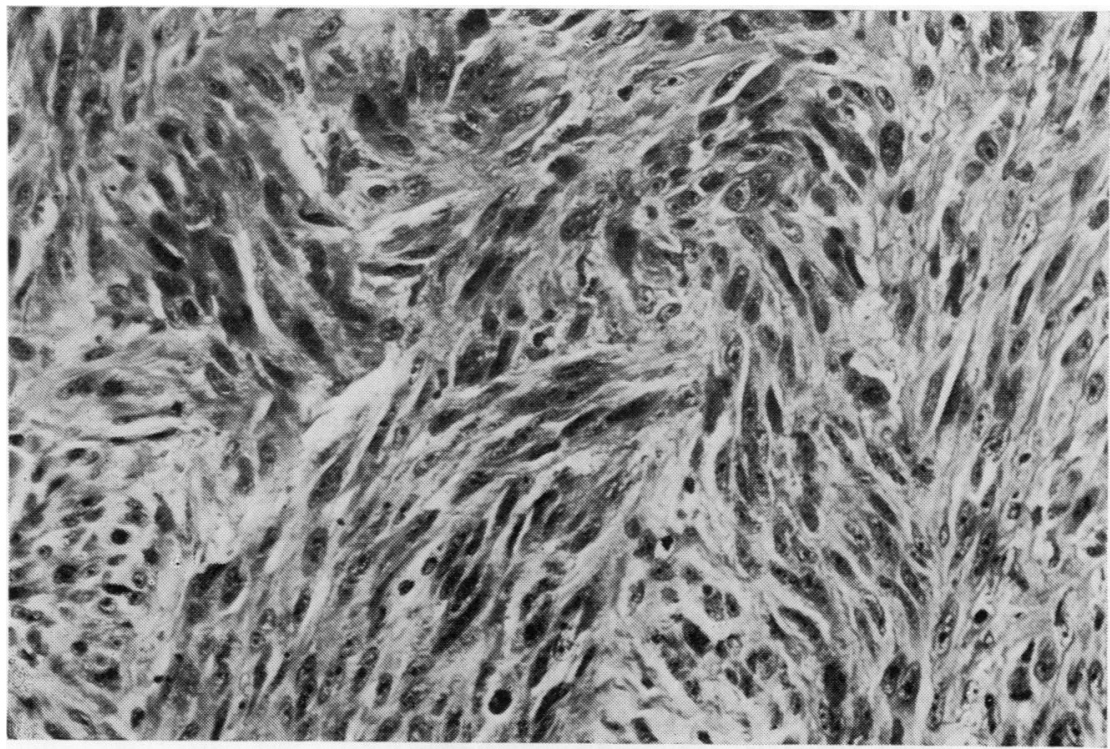

Fig. 15 Achromic spindle cell melanoma has been misinterpreted as fibrosarcoma, fibroxanthoma, and anaplastic squamous cell carcinoma.

\section{Invasive melanoma}

Invasive malignant melanoma is a polymorphous tumour (Figs 13-16) which, apart from showing melanogenesis, may assume naevic, epithelioid, and spindle cell forms with alveolar, acinar, fasciculated, and whorled growth patterns. Melanomas arising in association with lentigo maligna are often spindle cell in nature. Where all the histological features and growth patterns of a cutaneous tumour are those of melanoma, but pigment is absent, the tumour has the same biological potential as the common pigmented type and is referred to as amelanotic or achromic melanoma. When metastatic achromic tumour in a lymph node has the cellular characters of melanoma, the appearance should not be called more than suggestive of melanoma if there is no clinical evidence of a primary melanoma.

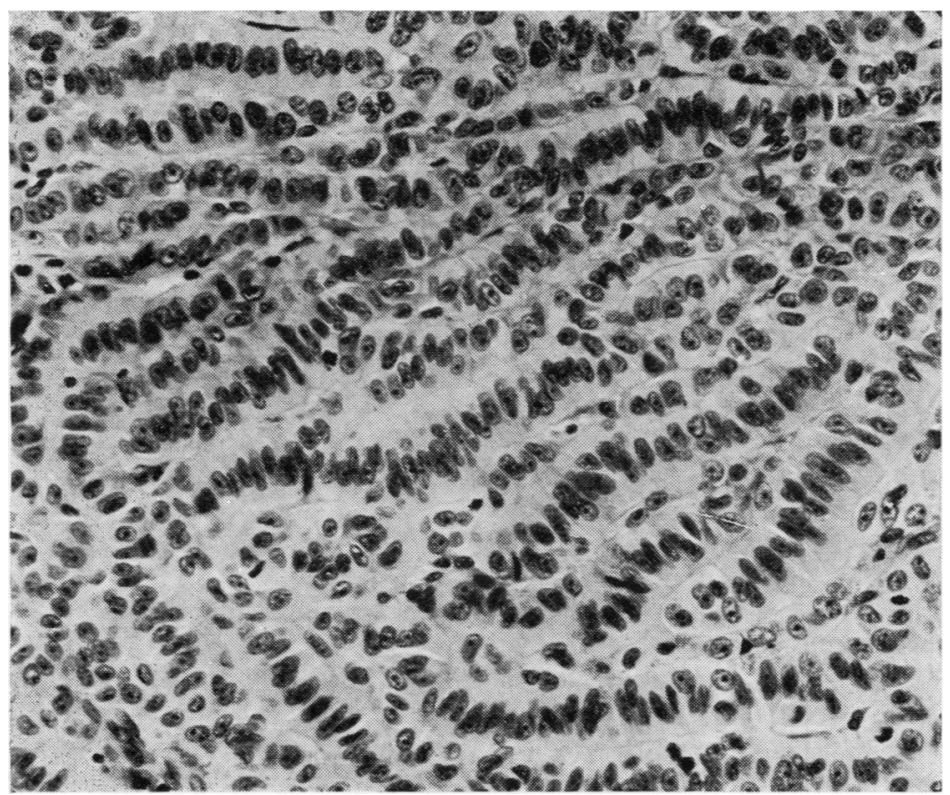

Fig. 16 A lymph node metastasis in which a gyrate trabecular pattern is conspicuous. 


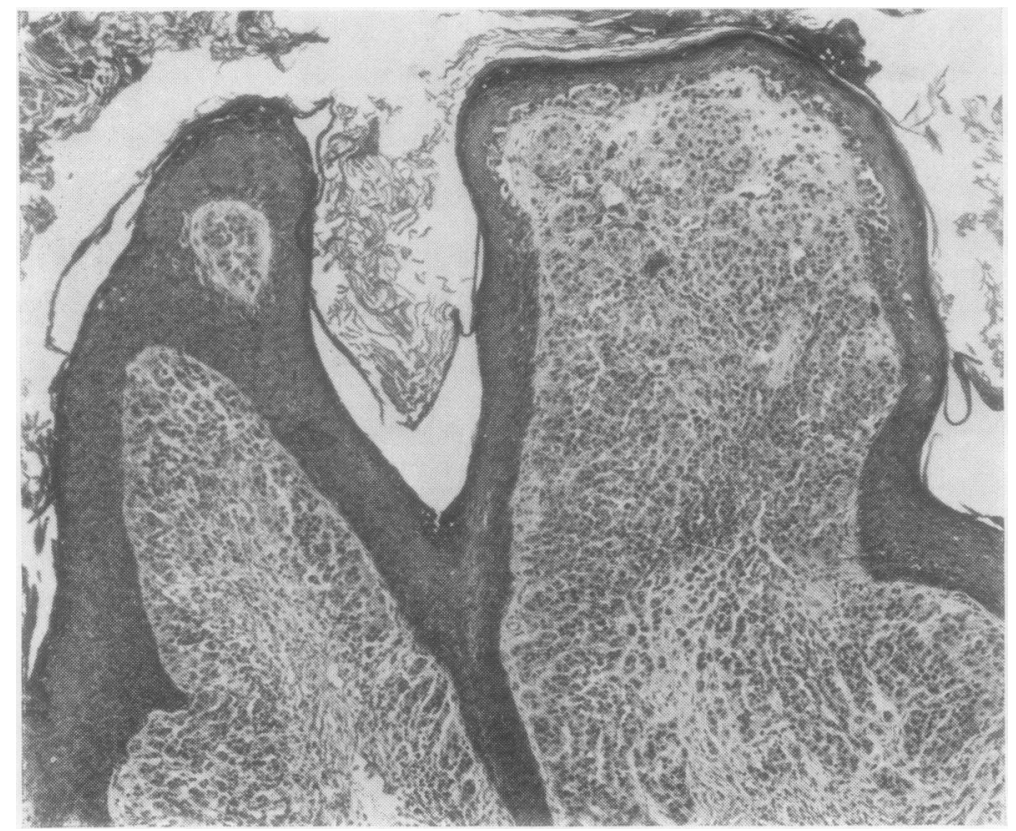

Fig. 17 A melanoma with a verrucose pseudonaevoid architecture.

It is important to distinguish the varieties of melanoma because their behaviour and the methods used in their management differ. Usually the distinction is readily made on clinical grounds. Lentigo maligna melanoma has a history of a waxing and waning pigmentation lasting perhaps for decades on the facial skin in almost all cases. When invasive tumour appears selective biopsies or preferably study of the whole lesion should provide the answer, but the changes may be equivocal. Appreciation of the difficulties of absolute distinction between superficial spreading and lentigo maligna melanoma at the light microscope level was the reason for theSydney group's including as group 3 in their classification of melanoma patterns 'malignant melanoma, invasive with adjacent intraepithelial component, unclassifiable' (McGovern et al., 1973). This group also recognised as a clinicopathological entity nodular melanoma characterised by rapid increase in size from its onset in either blemish-free skin or a naevus as a palpable, convex, frequently polypoid, and ulcerated tumour lacking pigmentation in the surrounding skin. In this type of tumour junctional change is rarely seen and, when present, does not extend to more than three rete pegs beyond the area of clear dermal invasion. Nodular melanoma carries the worst prognosis of the three common types of cutaneous melanoma.

A fourth clinical type of melanoma, not yet the subject of a serious description in the literature, may be described as verrucous and pseudonaevoid in type. The lesion is indolent clinically, resembles a warty intradermal naevus, and histologically has the structure of such a naevus (Fig. 17). However, close examination reveals that a part of the lesion is composed of cells that are slightly larger and more hyperchromatic than naevus cells and that mitoses are present in this seemingly innocent dermal infiltration (Fig. 18). There is even the appearance of nuclear crowding, interpreted as maturation in the depths of the naevus. This lesion is among the more difficult diagnostic pitfalls.

Recently described variants of melanoma include the balloon cell melanoma (Fig. 19) (Gardner and Vazquez, 1970; Hornstein, 1966) and a desmoplastic melanoma (Fig. 20) (Conley et al., 1971). Foci of balloon cell change and desmoplasia are features of otherwise typical melanoma, but in the variants so-named all or virtually all of the lesion exhibits these features. Balloon cell melanoma has been reported as a sebaceous gland or other primary skin tumour, hypernephroma, chondrosarcoma, and the desmoplastic melanoma as recurrent fibromatosis or fibrosarcoma.

\section{Secondary changes in malignant melanoma}

Malignant melanoma is noted for the appearances of secondary changes in the primary tumour interpreted as stromal, reactive, or retrogressive. The primary appearances may not be those of simple 


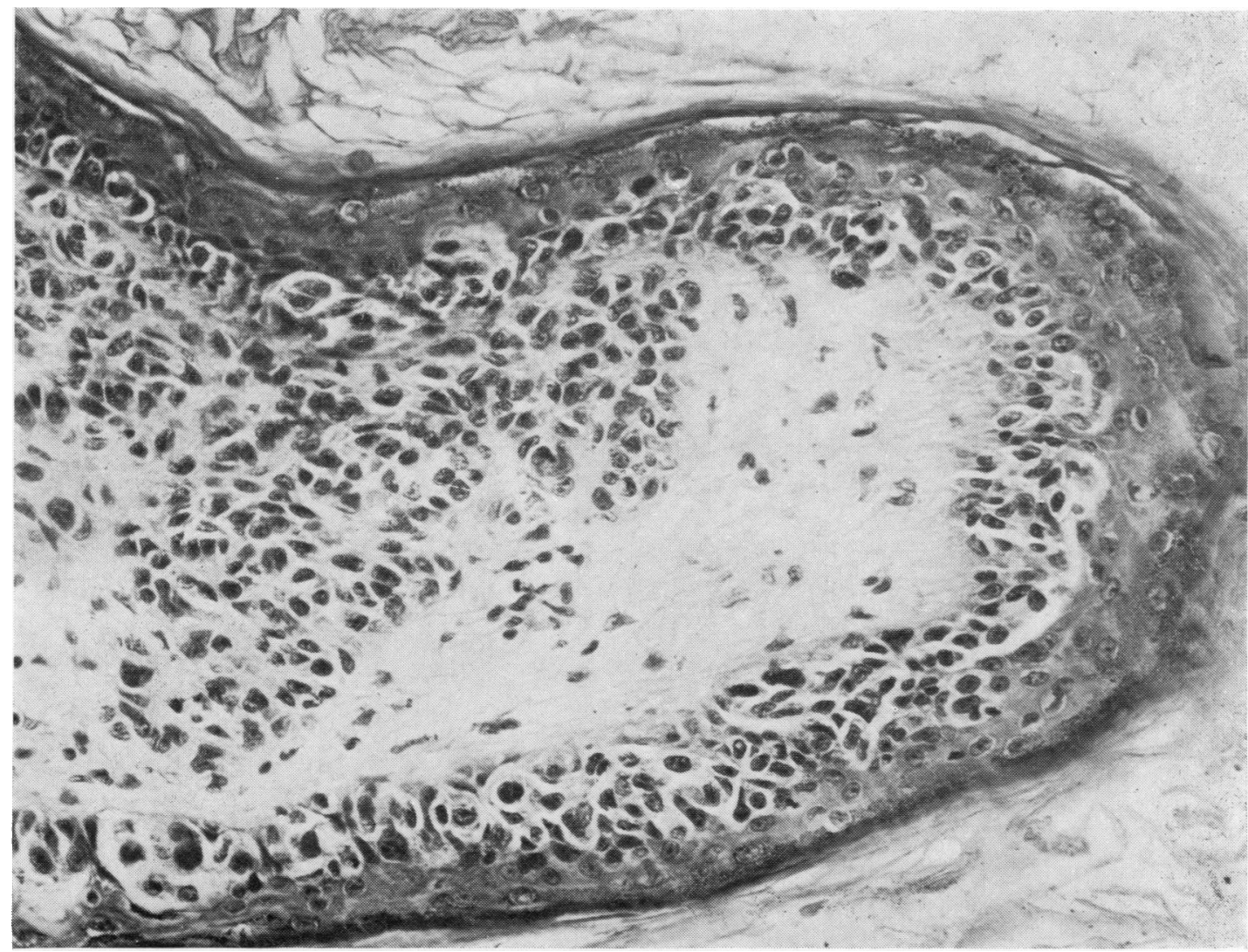

Fig. 18 A further example of a verrucose, pseudonaevoid melanoma under a higher magnification. The microarchitecture does not differ from that of a verrucose compound naevus, but two features distinguish melanoma from naevus: the continuous, as opposed to focal junctional change, and large, hyperchromatic nuclei with mitoses.

expansile or infiltrative growth which characterise other types of malignancy. Ulceration, hyperkeratosis, and papillomatosis may be seen with recognisable histological counterparts to these clinical features (Fig. 21). However, ulceration of a melanocytic proliferation, common in established melanoma, is not in itself indicative of malignancy. Trauma, isolated or repeated, is one cause, while cellular proliferation beneath an attenuated epidermis as in the Spitz naevus is another. Melanin in the keratinocytes and keratin, indicative of cytocrinia, is not of diagnostic value vis-à-vis innocence or malignancy, merely indicating that both benign and malignant tumour melanocytes may function in the same manner as normal melanocytes.

It is occasionally a European practice to excise a suspicious melanoma after a few days' irradiation. Post-irradiation appearances range from total disappearance of the lesion, when the diagnosis can reasonably be confirmed only if metastases become available for study, to partial or complete survival of apparently unaffected tumour. Another result is that of gross irradiation change with bizarre nuclear damage (Fig. 22). These are quite unlike the changes commonly found in basal cell carcinoma and squamous cell carcinoma after a more prolonged fractionated course of irradiation to a lower total dosage.

\section{Differential diagnosis of cutaneous malignant melanoma}

The naevocytic naevi are certainly the commonest lesions to be confused with melanoma among a large number of neoplastic as well as non-neoplastic proliferations, the complete list being co-extensive, probably with a text on dermatopathology (Belisario, 1966). After naevocytic naevi, blue naevi and the Spitz naevus are the benign proliferations most often mistaken for malignant melanoma. Less frequently, 


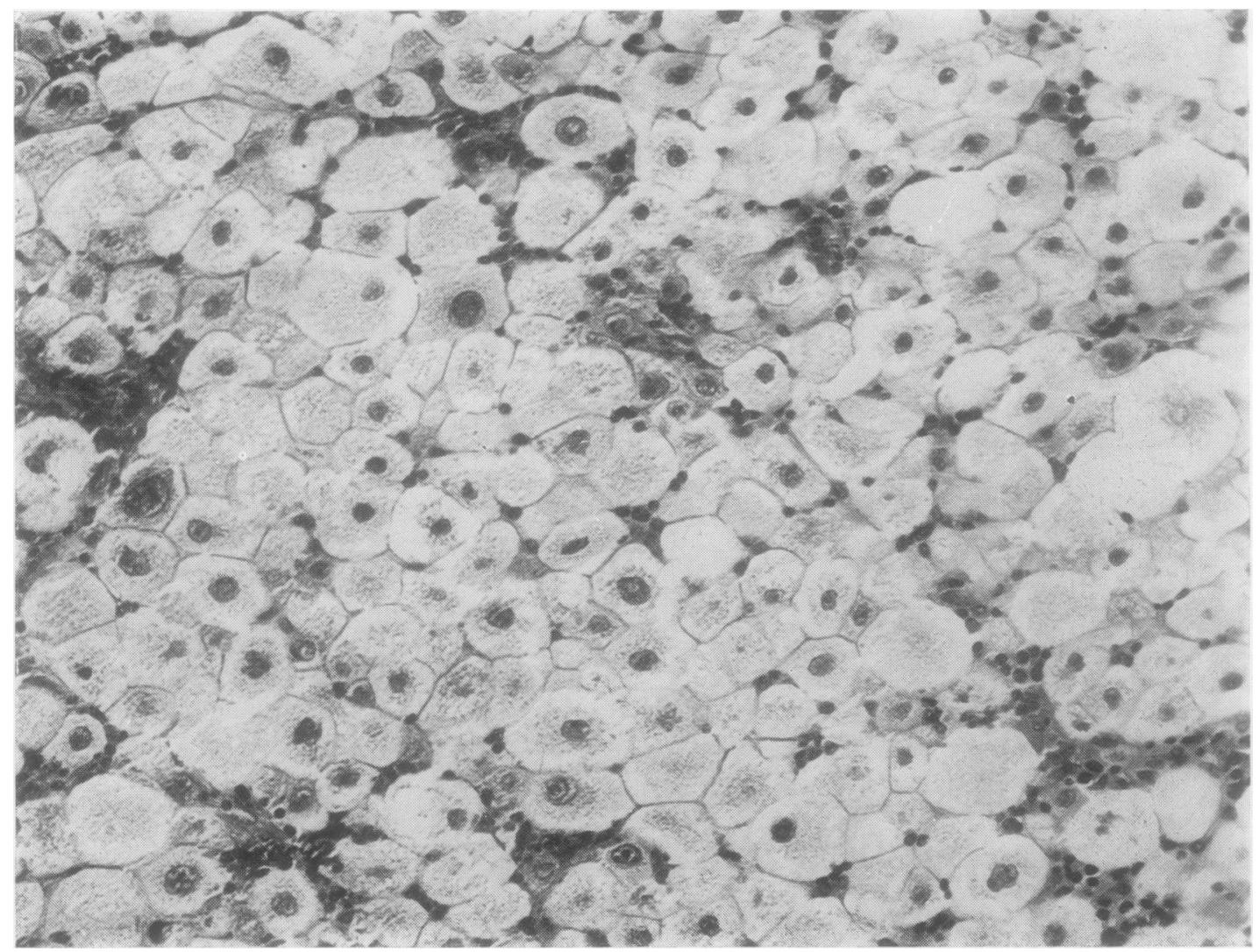

Fig. 19 Balloon cell melanoma. This may be misinterpreted in the primary lesion as a sebaceous gland carcinoma and in metastases as a clear cell carcinoma of renal, adrenal, or pulmonary origin. Balloon cells may be faintly $P A S+$ (diastase resistant) and slightly positive for acid mucopolysaccharides with Alcian blue. Pigment granules, when present, stain for melanin. In the primary tumour junctional change and melanin should be sought.

spindle cell carcinoma, squamous carcinomas, or basal cell carcinomas are similarly misdiagnosed. The benign pigmented lesions merit further discussion.

\section{Blue naevus}

Good accounts of the blue naevus are given by Lund and Kraus (1962) and Dorsey and Montgomery (1954). It is one of the commonest benign lesions to be misdiagnosed as melanoma. The blue naevus is a dermal melanocytoma, which occurs particularly on face and extremities and does not involve the epidermis in its growth, nor do the cells resemble ordinary naevus cells. They are larger, spindle-shaped, bi-polar, and dendritic, and are frequently grouped in the lower two-thirds of the dermis (Fig. 23). It is common for some degree of innocent junctional change to be present in association with a blue naevus, but whether a degree of junctional change is present or not there is a gap between epidermis and the blue naevus without continuity between epithelium and the melanocytic proliferation. Occasionally a true compound naevus is also present, and this combination tumour is likely to simulate the appearance of a melanoma clinically (Fig. 24). It is noteworthy also that among the very few areas of over-diagnosis that Little (Davis and Little, 1974) made in his great series of frozen section reports was one such combination lesion of a 'true with blue' naevus. Mitotic figures and abnormal nuclear forms are not rare in the variant termed cellular blue naevus (Fig. 25) and they are not in themselves an indication of malignancy. A notable feature of blue naevus in comparison with a malignant melanoma is the manner in which the naevus cells infiltrate the adnexa. These, though engulfed by melanocytic proliferation, are well preserved. 


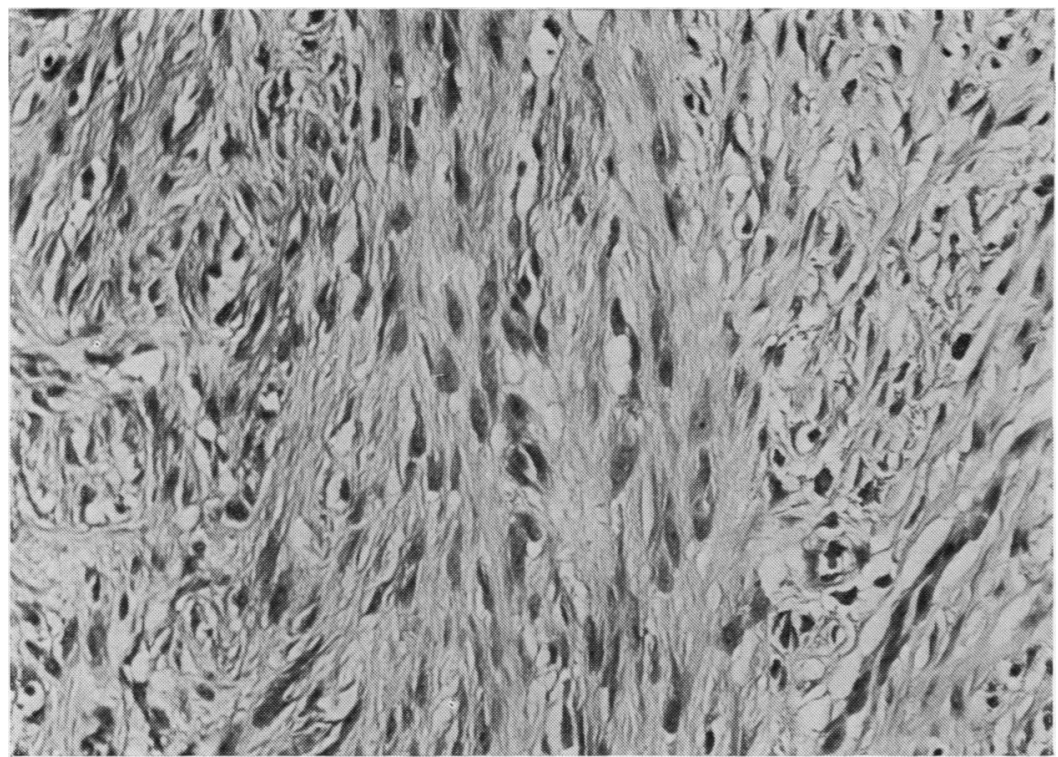

Fig. 20 The desmoplastic variant of melanoma is a diagnostic trap. Fibroxanthoma, fibroma, or fibrosarcoma are simulated by this tumour, which can be diagnosed when continuity of overlying junctional change with melanogenic tumour is demonstrable. There may be one of the commoner patterns of melanoma present merging with the desmoplastic tumour, when the diagnosis presents very little difficulty. A pigmented histiocytoma is occasionally associated with an overlying naevus. The discontinuity of the two lesions ant the demonstration that the dermal pigment is iron-containing suffice to differentiate this innocent collision tumour from desmoplastic melanoma.

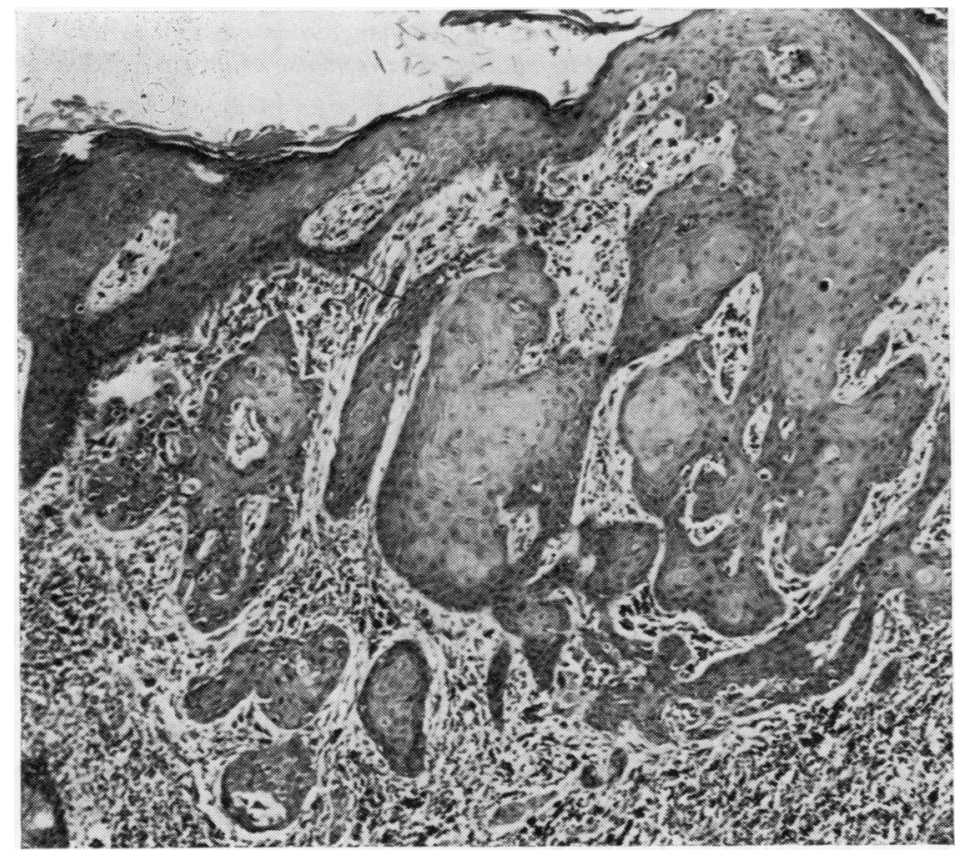

Fig. 21 This degree of pseudoepitheliomatous hyperplasia seen in a regressing melanoma is of clinical significance when it forms the major portion of a biopsy and its nature is misjudged. 


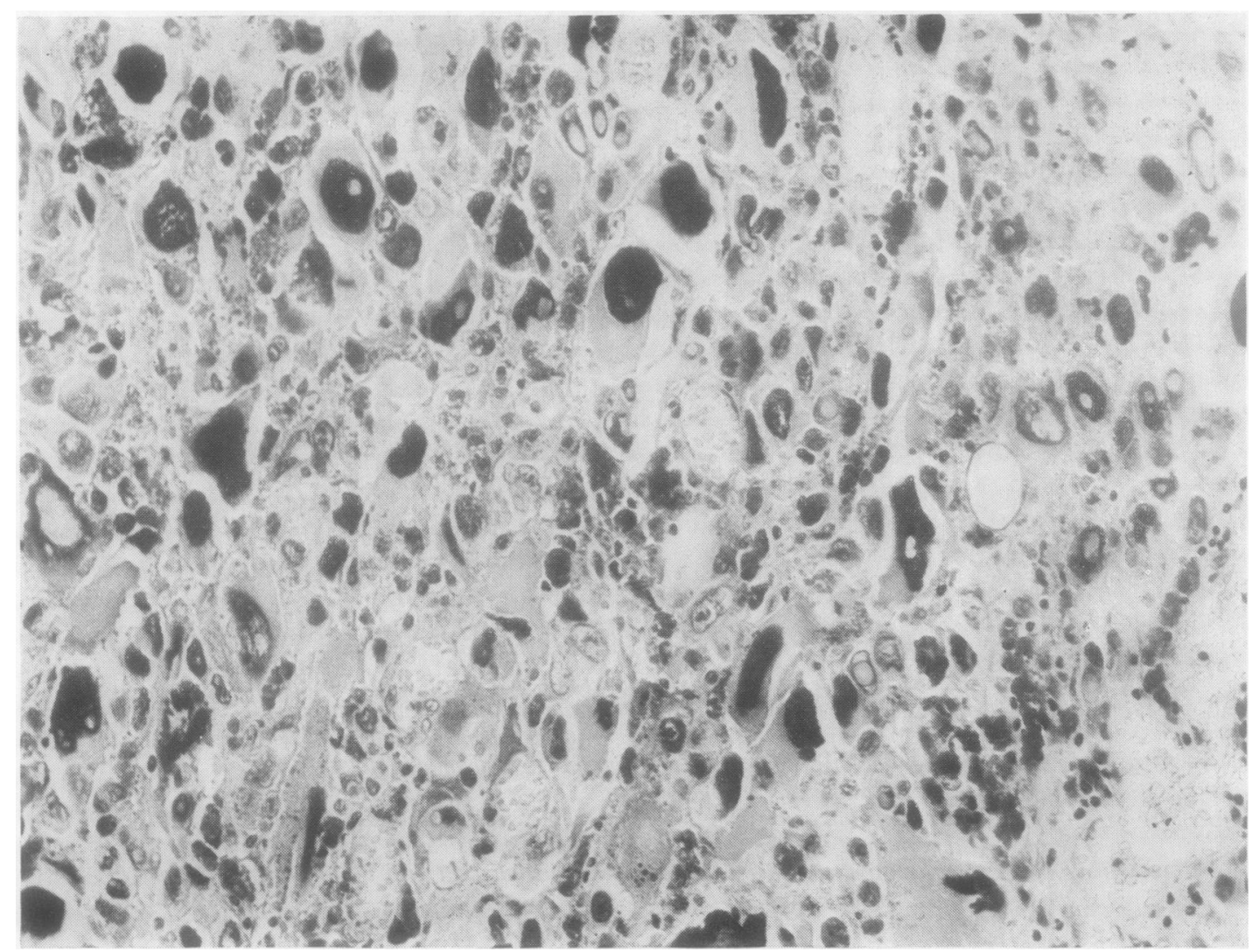

Fig. 22 Gross post-irradiation change in a primary melanoma. Lentigo maligna change responds to a variety of physical and chemical agents, eg, cryotherapy, topical $5 \mathrm{FU}$ and irradiation. The response of invasive melanoma to irradiation is unpredictable.

Malignant melanoma arising in a blue naevus or a melanoma whose overall architecture is that of a blue naevus is of excessive rarity and should not be diagnosed merely because in a spindle cell melanoma of nodular configuration no overlying or adjacent junctional change is demonstrable. The clinical criteria for the diagnosis of this tumour are:

1 a clinical background indicating the tumour is primary,

2 a growth pattern of blue naevus,

3 cellular anaplasia, and

4 the presence of necrosis.

A small intradermal deposit of metastatic melanoma, particularly when heavily pigmented spindle cell in type and involving adnexa, simulates a blue naevus. Attention to the history of the case, the location of the lesion, and a study of the section may also reveal the remains of a naevus or melanoma. A cellular blue naevus may have a recent history of rapid increase in size in a long-standing blemish, even to the development of pedunculation.

\section{Spitz naevus}

The Spitz naevus (synonyms: juvenile melanoma, epithelioid and spindle cell naevus) has a history and clinical presentation which differ from those of both melanoma and ordinary naevi (Kernen and Ackerman, 1960) (Figs 26-28). Its most frequent occurrence is in the first decade, but it may be encountered in old age. The face is a favourite site, and the lesion is pale and dome-shaped. Ulceration and bleeding are not infrequent. Histological features distinguishing it from melanoma are:

1 the junctional change has a scalloped or bitten-out appearance, and the epidermis is attenuated over the lesion rather than destroyed by 'Pagetoid' change; 


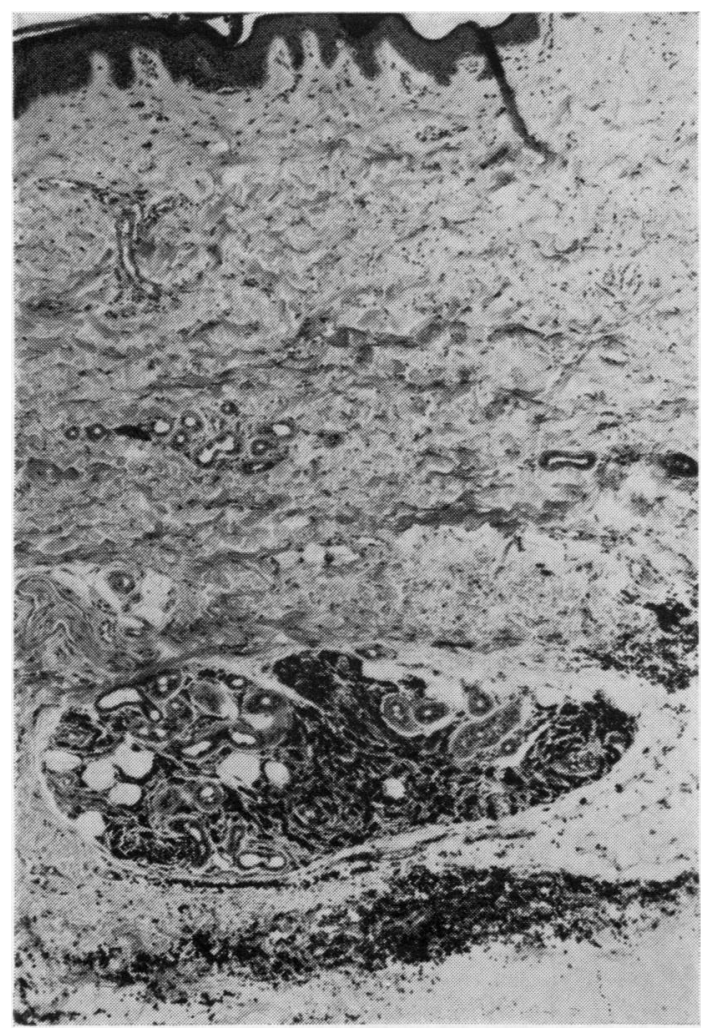

Fig. 23 A minute blue naevus restricted in distribution to a sweat gland coil. Clinically it was suspected of being a metastasis from a melanoma. Note the depth of the lesion and the preservation of the adnexa.

2 superficial oedema and telangiectasia may be present;

3 nucleoli present are not of the large owl's eye variety seen in melanoma;

4 deep maturation may be present along with fibrosis; and

5 the presence of characteristic giant cells.

Other characteristic features seen in these naevi are a 'raining down' appearance in which the spindle cells are arranged perpendicularly to the skin surface and the naevus cells appear to be set in the stroma rather than manifesting invasion and disruption of the collagen (Wayte, 1971). Mitoses, even in the occasional abnormal form (Kernen and Ackerman, 1960), and foci of nuclear pyknosis are not in themselves indicative of malignancy in a lesion otherwise considered to be a typical Spitz naevus.

The diagnosis of Spitz naevus should never be hazarded on an incisional biopsy fragment. Where the whole lesion is available for study there is usually no difficulty in correct evaluation. Sometımes a focus of juvenile melanoma change is seen in a compound naevus.

\section{Changes in lymph nodes and soft tissues}

Certain features relevant to the diagnosis of metastatic malignant melanoma in lymph nodes are important:

1 the appearances of metastatic melanoma,

2 pigment deposits in reticuloendothelial cells (melanin tattooing), and

3 innocent deposits of melanocytes within a lymph node.

In metastatic malignant melanoma no resemblance is to be expected between the appearances of the metastatic and primary tumours. It is unwise to assume the presence of a second, non-melanomatous, primary tumour because a lymph node metastasis, which appeared after excision of an invasive melanoma, is achromic (Fig. 19).

Pigment deposits in reticuloendothelial cells may arise with melanin transportation to the lymph nodes from a primary melanoma or result from the destruction of melanoma cells within the node. The differentiation of melanophages from melanoma cells is often a problem in frozen section diagnosis. The absence of cellular cohesion, the coarseness of the granules, and, above all, the innocent appearance of the nuclei in a bleached paraffin preparation characterise simple phagocytic activity.

Two forms of innocent deposits of melanocytes in lymph nodes are of clinical importance out of all proportion to their rarity, since ignorance of their occurrence is inevitably accompanied by the diagnosis of metastatic malignancy.

1 Groups of naevus cells of a type encountered in the compound and intradermal naevi (naevus cell naevi) are encountered by chance in lymph node groups associated with the limbs and the head and neck (Fig. 29). It is often a matter of conjecture whether there is any association with a mole within the regional lymphatic territory. The origin of naevus cells in lymph nodes is obscure, and their clinical behaviour is one of total quiescence.

2 Blue naevus cell deposits in lymph nodes (Fig. 30) are associated with a regional blue naevus of any type in the author's experience. They may grow very slowly over decades. Their clinical presentation is either as an incidental finding or in lymph nodes removed deliberately in the management of a large blue naevus judged, erroneously, to be malignant on histological grounds. Though they may grow slowly, further metastasis does not occur, and in practice they must be considered to be a biologically benign or non-aggressive tumour form (Gricouroff et al., 1974). These deposits of blue 


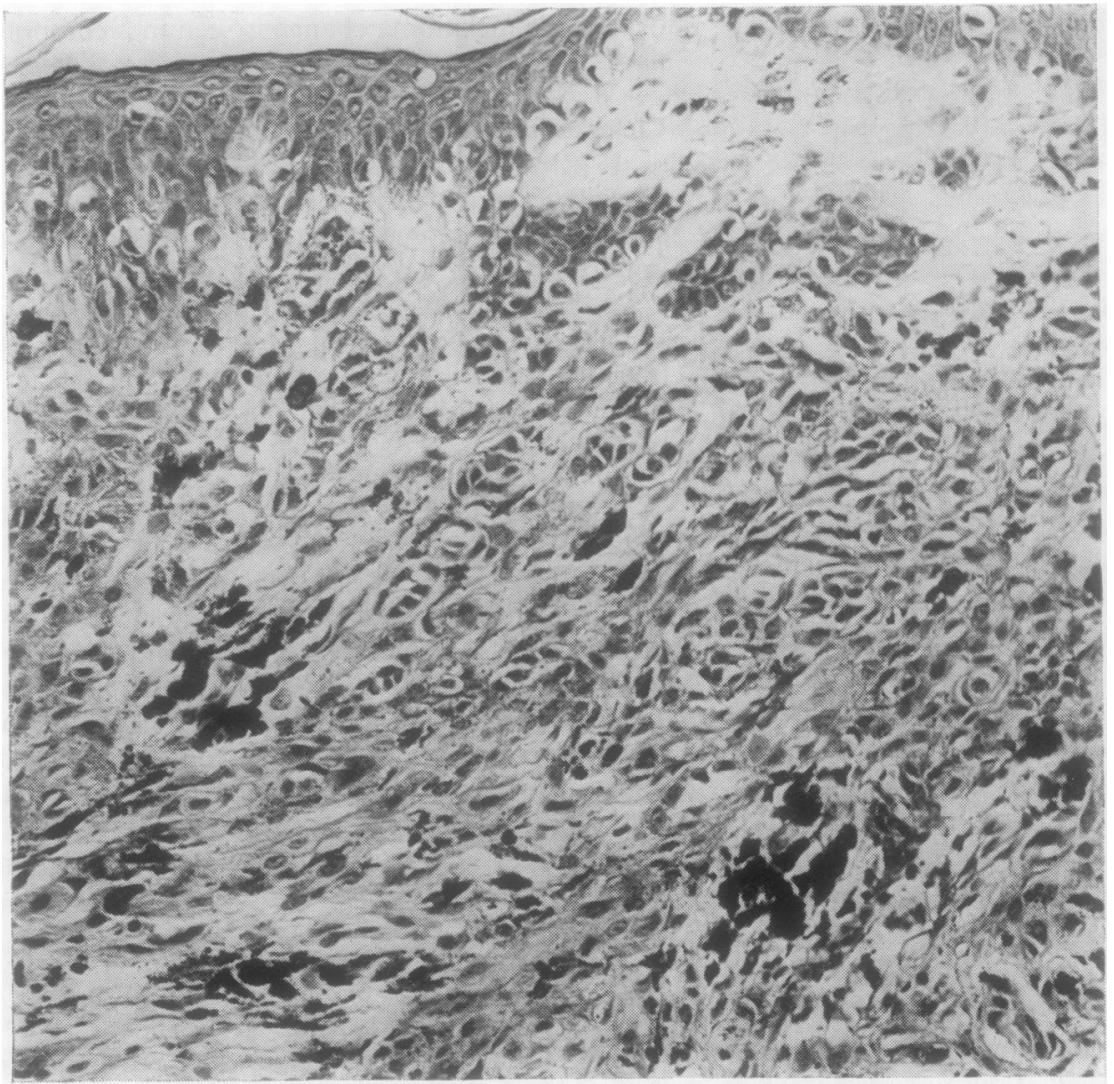

Fig. 24 Commonly. mistaken for melanoma is the combination of blue naevus with overlying junctional change, and the differentiation may be difficult on frozen section. The quality of the junctional change, the presence of a grenz zone, and the obvious blue naevus quality of the dermal infiltration are distinguishing features.

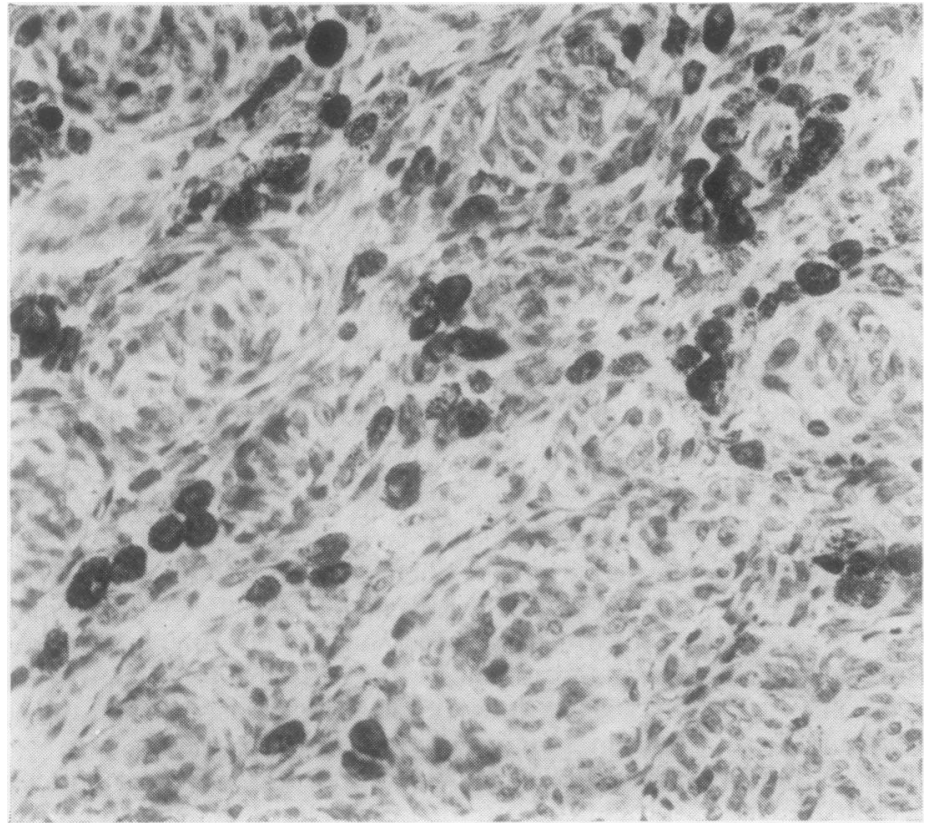

Fig. 25 A frequently seen pattern of the cellular blue naevus is of whorled balls of pale cells with heavily pigmented macrophages scattered in the interstices. 


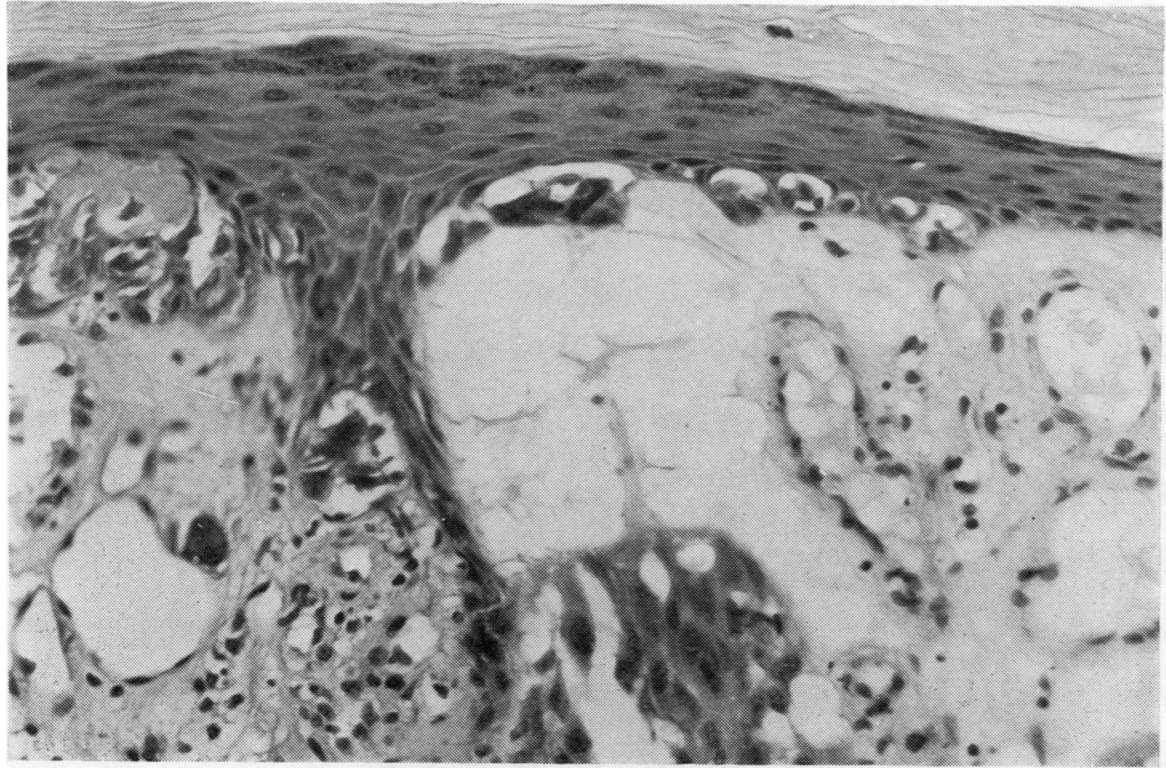

Fig. 26 Oedema and telangiectasia in the papillary dermis are notable features of some juvenile melanomas. The destructive junctional change remains basal and has a scalloped quality to it.

naevus are trabecular and peripheral in their distribution within the lymph node.

In soft tissues the recently described clear cell sarcoma may be readily confused with malignant melanoma. The diagnosis of clear cell sarcoma of tendons and aponeuroses (Enzinger, 1965) of either achromic or melanotic type requires great circumspection when there is a history of previous excision of melanoma of skin or other primary site. For the present, no sure distinction can be made between this tumour and some examples of metastatic melanoma. Another soft tissue melanotic tumour that has been confused with metastatic melanoma is the entirely benign pigmented schwannoma. In both of these cases the clinical importance attached to their being correctly diagnosed far outweighs a consideration of the great rarity of the lesion.

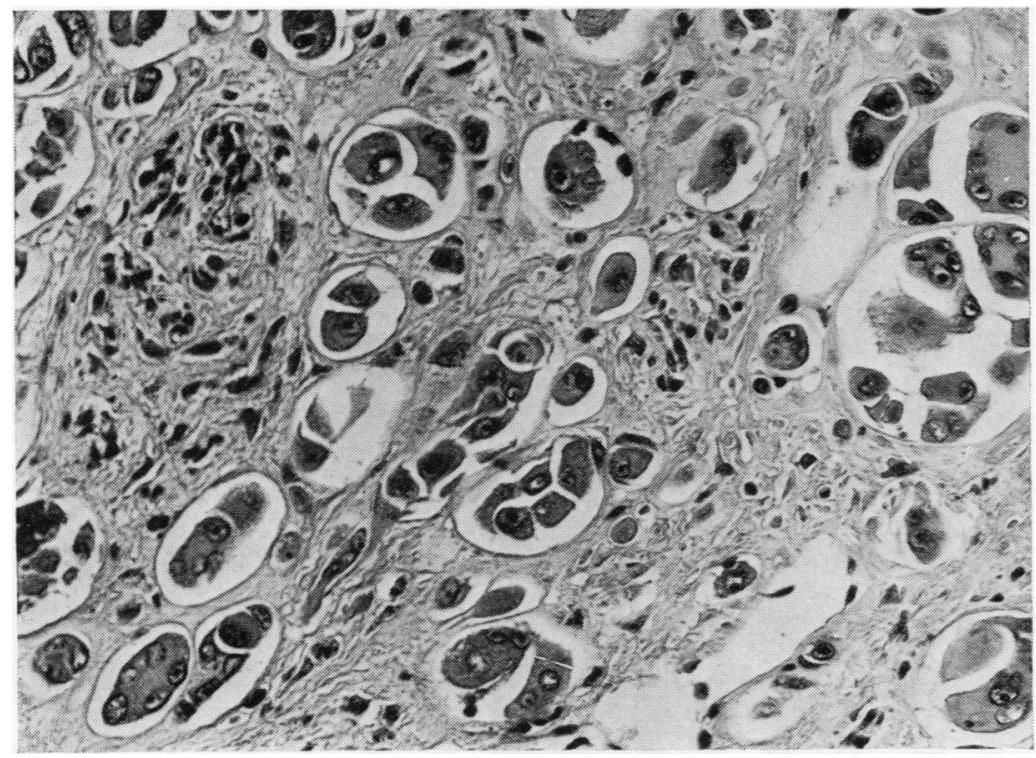

Fig. 27 Multinucleated, brightly acidophilic giant cells scattered with an alveolar grouping in a fibrous stroma are a feature of the epithelioid type of juvenile melanoma. An infiltrating melanoma is a cohesive tumour and, unless regressing or of desmoplastic type, devoid of fibrous interstitium. Artifactual shrinkage of epithelioid naevus cells away from their stromal surround is a distinctive histological appearance. 


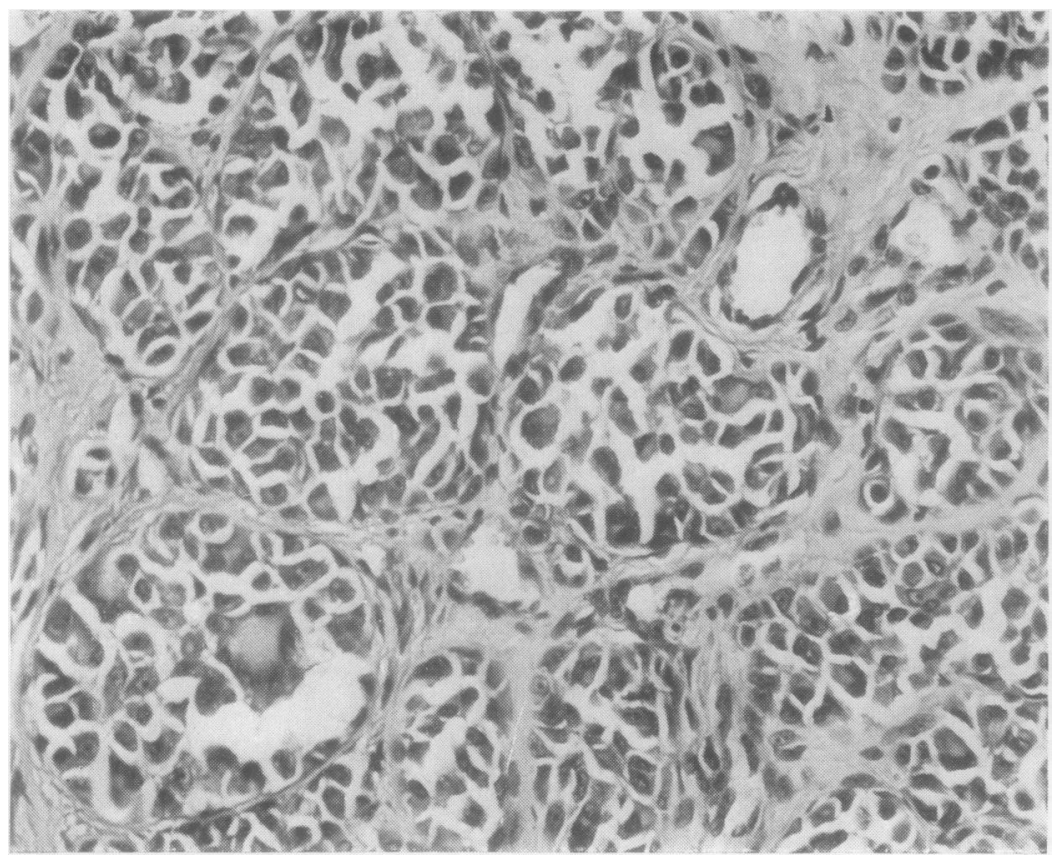

Fig. 28 A juvenile melanoma with an alveolar growth pattern. Vacuoles and apparently. phagocytosed cells are occasionally seen in the cytoplasm of the giant cells in juvenile melanoma.

\section{Prognosis of malignant melanoma}

It is important that the pathologist provides as much guide to prognosis as is available from his study of the material.

Good prognostic features in primary melanoma are a tumour of small dimensions (less than $0.75 \mathrm{~mm}$ thick and superficially invasive, as assessed by

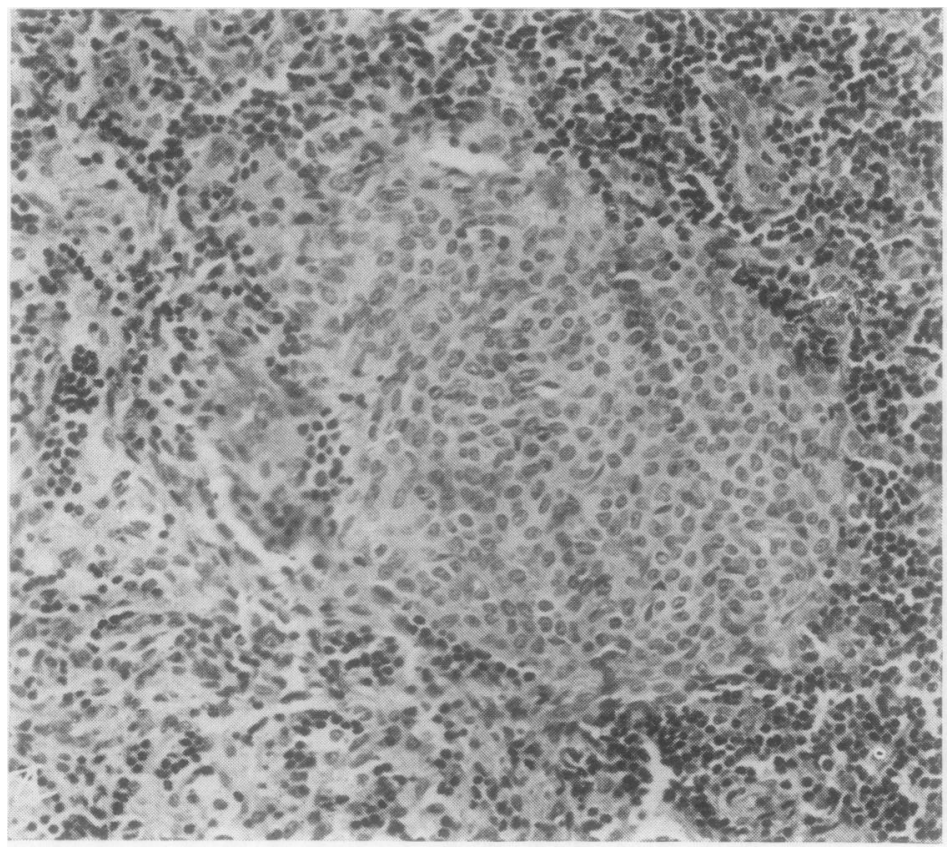

Fig. 29 A naevus cell deposit in a lymph node. This is a rare occurrence but most frequently encountered in axillary dissections carried out for an unrelated disorder, eg, mammary carcinoma. 


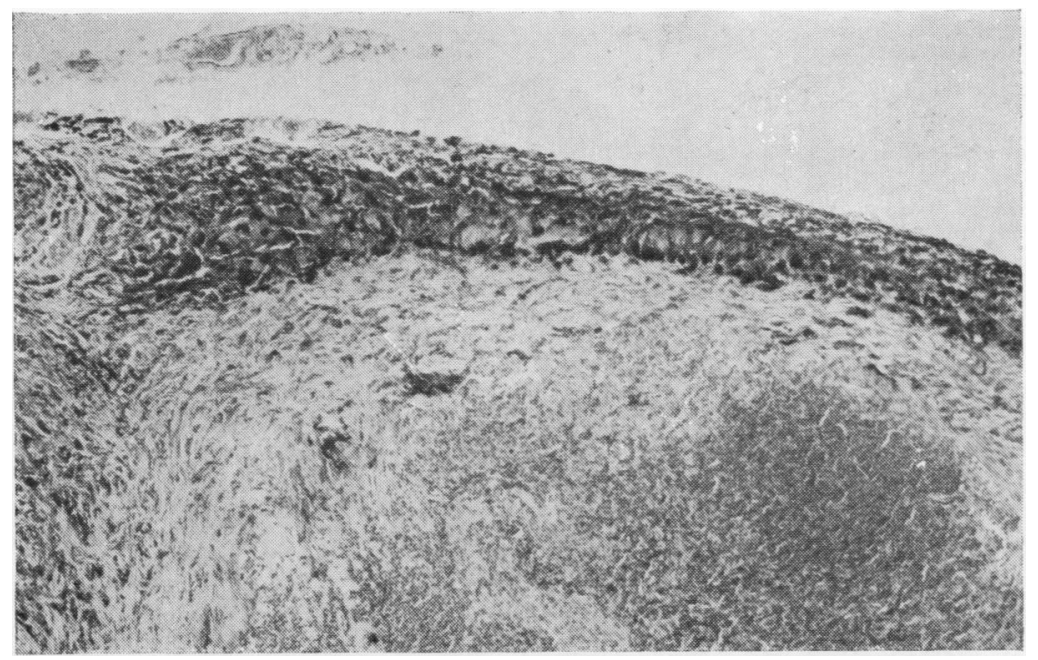

Fig. 30 Blue naevus deposits in lymph nodes are characteristically subcapsular, extending into the middle of the node via fibrous trabeculae. The thin dark rim to the node seen under the scanning lens is virtually diagnostic.

Clark's method (Clark et al., 1969)) and situated on the head and neck or lower extremities. Bad prognostic factors are a situation on the trunk, a polypoid or ulcerated lesion, and, of course, lymphatic or haematogenous dissemination. The outlook is worse in males. There is no association of any cytological type with a specific behaviour pattern or prognosis, nor indeed any constant replication of the principal growth pattern of the primary tumour in the metastases. Reports on the prognostic significance of any associated inflammatory infiltrate are conflicting.

The only consistent histological guide to prognosis is the depth of the invasion of the melanoma at its maximum thickness (Breslow, 1975). When the change is in situ, as in Hutchinson's freckle and some cases of superficial spreading melanoma, there is virtually no mortality. With invasion of the subcutaneous fat the 10-year survival rate is of the order of $10 \%$, and with intermediate levels there is a proportional mortality. There are some anomalies in the use of staging by histological level of invasion (Levene, 1975) but Breslow has cut across these problems by convincingly demonstrating that the maximal thickness of the lesion '... measured in several slides from the top of the granular layer to the deepest point of invasion ... or from the ulcer base to the deepest point of invasion' is a better measure of prognosis than is the level of invasion of the tumour. The chance of developing recurrent melanoma appears to be directly proportional to tumour thickness, and with lesions less than $0.76 \mathrm{~cm}$ thick there was no recurrent disease at five years. This paper and its successor, 'Optimal size of resection margin for thin cutaneous melanomas' (Breslow and Macht, 1977), are the most important contributions to the management of melanoma published in the last 20 years.

There is no improvement of prognosis resulting from wider, compared with narrower, excision (Olsen, 1966) as measured by the ensuing incidence of local recurrence, and there is no literature relating wide excision to overall length of survival. Surgeons need informing that a $2 \mathrm{~cm}$ clearance is probably quite adequate overall, that a centimetre is adequate for small thin melanomas, and that massive skin ablation serves no therapeutic function. The desirable breadth of excision for cutaneous melanoma is a proper subject for surgical research. The review of the whole subject by Breslow (1978) is comprehensive.

\section{Spontaneous regression of melanoma}

Irrespective of histogenesis, melanoma is prone to spontaneous regression in some degree. Those referred to simply as 'spontaneously regressing melanoma' are ones in which such disappearance of tumour has occurred that only a portion of negligible dimensions persists (Fig. 31). Spontaneous regression may present in three ways:

1 as the patient's testimony to the disappearance of the whole or part of the tumour at some stage of its evolution, 


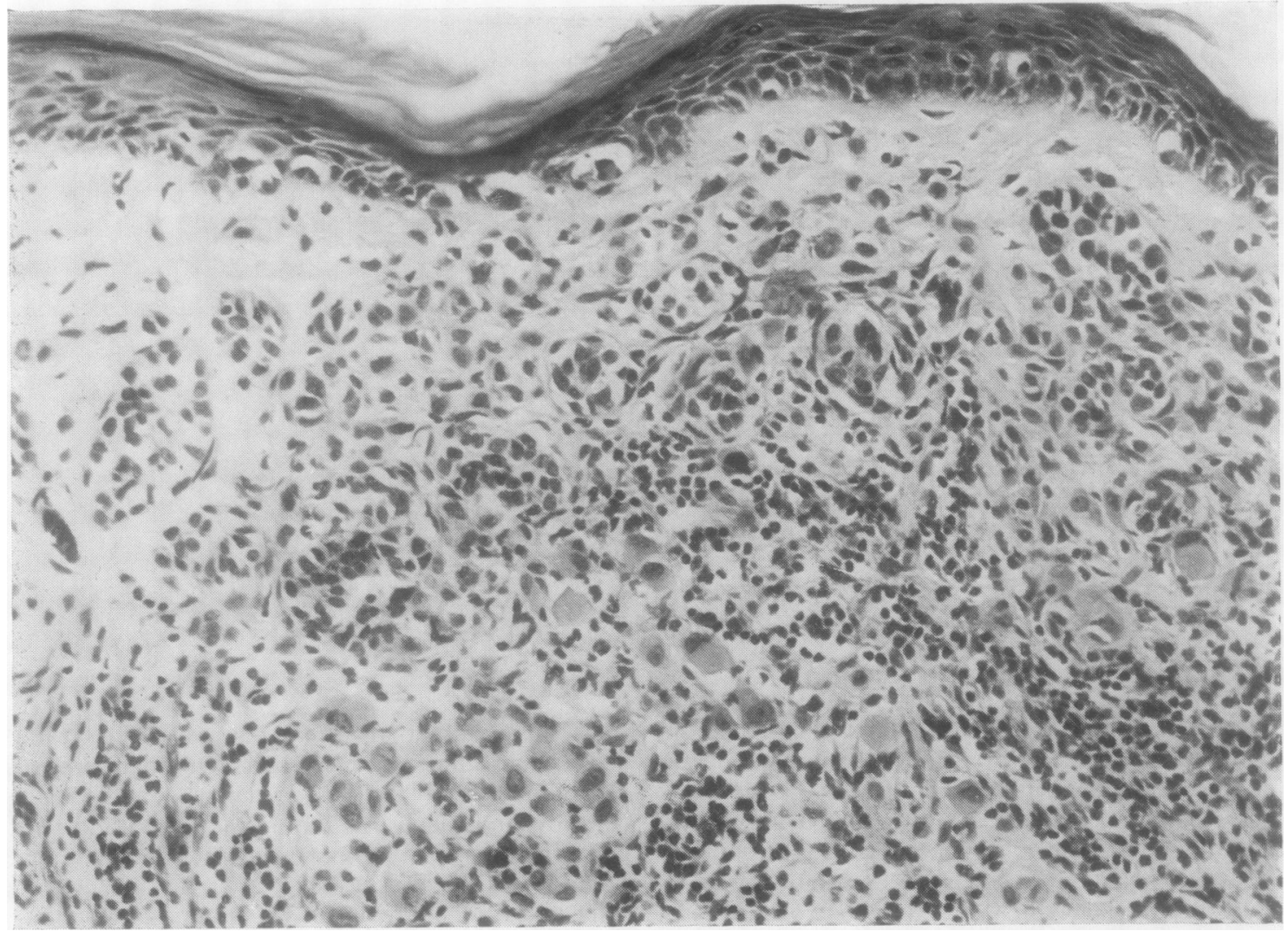

Fig. 31 Atypical basal melanocytes in the epidermis and overlying a regressing melanoma. A field no larger than this may be all that is discovered in a surgical specimen of a regressed melanoma. The clinician is led to detecting the small, usually vitiliginous area as the result of questioning the patient after the excision of a metastatic node.

2 as a histological finding, or

3 as occult melanoma, when a patient presents with regional lymph node metastases, and the site of the regressed primary tumour is identified by clinical means.

Histological evidence of regression is well summarised by McGovern et al. (1973): 'In the early stages there is a heavy lymphocytic infiltrate with pigment-containing macrophages. The outlines of affected melanocytes become indistinct and the cells disintegrate. As the process subsides, the lymphocytic infiltrate diminishes and an area of fibrosis with increased vascularity is left. The process may affect only a portion of a tumour and then subside completely having destroyed, either completely or in part, the tumour cells in that region.' These changes of regression have no prognostic significance.

Caution should be exercised in assessing the depth of invasion of melanomas exhibiting spontaneous regression. When this change is present the dimi- nution in tumour volume may be reflected in a loss of depth of invasion which is only apparent. Anomalous behaviour of some melanomas considered as thin is explicable on this basis.

The distinction from a regressing mole may be difficult for there may be no difference between the two conditions in the histories and the physical findings. In the histological appearances there may be few identifiable cells other than inflammatory ones and melanophages in the dermis, and the little junctional changes present may be of equivocal status. In these cases the vascularity of the regressed or regressing melanoma is the major distinguishing feature. Usually, however, the junctional cells as well as the scattered invasive cells appear to be melanomatous, not naevoid, in character. Depigmentation of the overlying epidermis, a feature of both regressing ordinary naevus and melanoma, is not, to my knowledge, seen in any stage of the Spitz naevus, with which some macrocytic melanomas have a 
resemblance, particularly when regressing, but this belief merits investigation.

\section{Appendix}

NOTES ON DIAGNOSTIC METHODS IN

CUTANEOUS MELANOMAS

The history of the lesion should always be available to the pathologist. A casual, uninformed approach may lead, for example, to confusion of blue naevus with a satellite nodule of spindle cell melanoma, of primary and secondary melanoma and Spitz naevus with achromic melanoma. The recently delineated clear cell sarcoma of tendon sheaths (Enzinger, 1965) should not be diagnosed when there is a history of excised melanoma.

Surgical specimens containing melanoma are of three types: small, incisional biopsies, narrow total excisions, and definitive wide excisions. Incisional biopsies (Epstein, 1971; Davis and McLeod, 1972) may not be representative. Pseudoepitheliomatous hyperplasia (Fig. 21) or simple naevus only may be seen in the biopsy while the associated melanoma is unrecognised. The diagnosis of some benign melanocytomas such as the Spitz naevus, cellular blue naevus, and the halo naevus of Sutton depend on an assessment of the overall microarchitecture. Moreover, depth of melanoma invasion cannot be assessed on such limited material.

Specimens are conveniently studied after adequate formalin fixation. Small specimens may be orientated by flattening on card, and larger specimens should be pinned on cork before fixation. A good line drawing of the specimen is recommended, and the dimensions of the lesion and its surrounds should be recorded. Depending on the size of the lesion, parallel slices should be cut and examined with the naked eye, and a selection of blocks made for paraffin embedding. The surrounding skin should be examined for nodules in dermis and subcutaneous fat by palpation and serial slicing.

Discretion should be exercised in the selection of tissue blocks. With small lesions less than $1 \mathrm{~cm}$ in diameter, the whole lesion should be processed and sections studied at progressively deeper levels as required. The practice of block selection used to study adequacy of clearance of epitheliomatous ulcers should not be applied to the diagnosis of melanoma since the procedures may be destructive and make diagnosis uncertain. With larger tumours, more blocks should be examined so that the maximal depth of infiltration may be ascertained. However, it is superfluous to study the perimeter of a $6 \mathrm{~cm}$ diameter skin excision around a superficial $1 \mathrm{~cm}$ lesion. In reporting on the melanoma narrowly excised for diagnostic purposes only, there is no point in commenting on the inadequacy of the excision. Such comment irritates our surgical colleagues (Levene, 1977).

Haematoxylin and eosin paraffin sections comprise the primary method of examination but they may need to be supplemented by a melanin bleach to allow visualisation of nuclei in heavily pigmented cells, by Fontana silver impregnation and Schmorl's reaction to detect or confirm the presence of melanin, or by Perls' acid ferrocyanide reaction to exclude iron pigmentation.

It is important that the histological techniques are of the highest quality so that inferior ambiguous preparations are not produced, and that diagnostically imporiant sections are examined. Unintelligent excessive trimming of small lesions may destroy evidence of a melanoma; conversely, inadequate examination of a block by using the first available section as the basis for the histological report may result in serious underdiagnosis.

\section{Frozen section diagnosis}

As with all other situations in which cryostat sections have to be interpreted, familiarity should lead to accuracy and confidence in practice. Where there is little familiarity with primary melanoma, it is unwise to employ the method. It is, however, standard practice in the major cancer centres now since depth of tumour invasion is taken into consideration when tumour excision and simultaneous regional lymphadenectomy is contemplated.

\section{Reporting}

The essential information to be derived from the preparation is the type of melanoma and its maximal depth of infiltration as well as the presence of lymphatic permeation. Other features which play a variable but subsidiary role in determining the final histological assessment include the presence of ulceration, evidence of a pre-existing naevus, the degree of pigmentation, the cytological appearances, and the extent and quality of the inflammatory response, together with evidence of spontaneous regression, and these should be recorded.

I thank those pathologists in England and Europe who have contributed to my understanding of melanoma by referral of problematic or interesting cases, the World Health Organisation for providing access to an unprecedented volume of pathological specimens, my secretary, Ruth Higgs, for preparing the manuscript, and, finally, my friend, Ken Moreman, FIIP, FRPS, for preparing the photomicrographs. 


\section{References}

Belisario, J. C. (1966). Malignant melanoblastomas; some usual and unusual aspects and vagaries. Australian Journal of Dermatology, 8, 224-231.

Breathnach, A. S. (1968). The epidermal Langerhans cell. British Journal of Dermatology, 80, 688-689.

Breslow, A. (1975). Tumor thickness, level of invasion and node dissection in stage 1 cutaneous melanoma. Annals of Surgery, 182, 572-575.

Breslow, A., and Macht, S. D. (1977). Optimal size of resection margin for thin cutaneous melanoma. Surgery, Gynecology and Obstetrics, 145, 691-692.

Breslow, A. (1978). The surgical treatment of stage I cutaneous melanoma. Cancer Treatment Reviews, 5 , 195-198.

Clark, W. H., Jr., From, L., Bernardino, E. A., and Mihm, M. C., Jr. (1969). The histogenesis and biologic behaviour of primary human malignant melanomas of the skin. Cancer Research, 29, 705-726.

Clark, W. H., Jr., and Mihm, M. C., Jr. (1969). Lentigo maligna and lentigo-maligna melanoma. American Journal of Pathology, 55, 39-67.

Conley, J., Lattes, R., and Orr, W. (1971). Desmoplastic malignant melanoma (a rare variant of spindle cell melanoma). Cancer, 28, 914-936.

Davis, N. C., and Little, J. H. (1974). The role of frozen section in the diagnosis and management of malignant melanoma. British Journal of Surgery, 61, 505-508.

Davis, N. C., and McLeod, G. R. (1972). The surgery of primary melanoma, problems and practice. The Medical Journal of Australia, 2, 778-782.

Dorsey, C. S., and Montgomery, H. (1954). Blue nevus and its distinction from Mongolian spot and the nevus of Ota. Journal of Investigative Dermatology, 22, 225-236

Enzinger, F. M. (1965). Clear-cell sarcoma of tendons and aponeuroses: An analysis of 21 cases. Cancer, 18, 1163-1174.

Epstein, E. (1971). Effect of biopsy on the prognosis of melanoma. Journal of Surgical Oncology, 3, 251-255.

Gardner, W. A., Jr., and Vazquez, M. D. (1970). Balloon cell melanoma. Archives of Pathology, 89, 470-472.

Gricouroff, G., Mazabraud, A., and Dulac, G. (1974). À propos d'un cas de naevus bleu avec envahissement ganglionnaire. Bulletin de Cancer, 61, 137-150.

Hornstein, O. (1966). Zur Kenntnis des sogenannten Blasenzellnaevus. Archiv für klinische und experimentelle Dermatologie, 226, 97-110.

Kernen, J. A., and Ackerman, L. V. (1960). Spindle cell nevi and epithelioid cell nevi (so-called juvenile melanomas) in children and adults. Cancer, 13, 612-625.

Kopf, A. W., and Andrade, R. (1963). A histologic study of the dermoepidermal junction in clinically "intradermal" nevi, employing serial sections I. junctional theques. Annals of the New York Academy of Sciences, 100, 200-220.
Kornberg, R., and Ackerman, A. B. (1975). Pseudomelanoma. Recurrent melanocytic nevus following partial surgical removal. Archives of Dermatology, 111, 1588-1590.

Levene, A. (1975). The diagnosis of malignant melanoma. In Proceedings of the XIth International Cancer Congress, 1974 (International Congress Series No. 354), edited by P. Bucalossi, U. Veronesi, and N. Cascinelli, vol. 6, pp. 89-93. Excerpta Medica, Amsterdam.

Levene, A. (1977). Selected topics in tumor pathology. In Principles of Surgical Oncology, edited by R. W. Raven. Plenum, New York.

Lund, H. Z., and Kraus, J. M. (1962). Melanotic Tumors of the Skin (Atlas of Tumor Pathology, Section 1, Fascicle 3). Armed Forces Institute of Pathology, Washington, D.C.

Lund, H. Z., and Stobbe, G. D. (1949). The natural history of the pigmented nevus; factors of age and anatomic location. American Journal of Pathology, 25, 1117-1155.

McGovern, V. J., Mihm, M. C., Jr., Bailly, C., et al. (1973). The classification of malignant melanoma and its histologic reporting. Cancer, 32, 1446-1457.

Masson, P. (1951). My conception of cellular nevi. Cancer, 4, 9-38.

Olsen, G. (1966). The malignant melanoma of the skin. Acta Chirurgica Scandinavica, Supplement 365.

Shaffer, B. (1955). Pigmented nevi. Archives of Dermatology, 72, 120-132.

Spitz, S. (1948). Melanomas of childhood. American Journal of Pathology, 24, 591-609.

Unna, P. G. (1896). The Histopathology of the Diseases of the Skin. Abstract from pp. 1137 and 1126. W. F. Clay, Edinburgh. Macmillan, New York.

Wayte, D. M. (1971) Pathology of nevi and melanomas. In The Skin, edited by E. B. Helwig and F. K. Mostofi, pp. 490-532. Williams and Wilkins, Baltimore.

\section{Additonal references}

The three following papers are likely to be very helpful. They are recommended for their comprehensiveness and balanced approach to the subject:

Kopf, A. W., Bart, R. S., and Rodriguez-Sains, Rene S. (1977). Malignant melanoma; a review. The Journal of Dermatologic Surgery and Oncology, 3, 41-125.

Paniago-Pereira, C., Maize, J. C., and Ackerman, A. B. (1978). Nevus of large spindle and/or epithelioid cells (Spitz's nevus). Archives of Dermatology, 114: 18111823.

Reed, R. J., Ichinose, H., Clark, W. H., Jr., and Mihm, M. C., Jr. (1975). Common and uncommon melanocytic nevi and borderline melanomas. Seminars in Oncology, 2, 119-147.

Requests for reprints to: Dr A Levene, Department of Histopathology, Royal Marsden Hospital, Fulham Road, London SW3. 